\title{
Computational Study on the Modification of a Bounding Surface Plasticity Model for Sands
}

D. M. G. Taborda ${ }^{a^{*}}$, L. Zdravkovic ${ }^{a}$, S. Kontoe ${ }^{a}$ \& D. M. Potts ${ }^{a}$

${ }^{a}$ Imperial College London, Department of Civil \& Environmental Engineering, Skempton Building, London SW7 2AZ, UK.

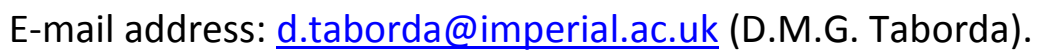

\section{Key words}

constitutive modelling, bounding surface plasticity, sands

\section{Abstract}

The accurate simulation of complex dynamic phenomena requires the availability of advanced constitutive models capable of simulating a wide range of features of soil behaviour under cyclic loading. One possible strategy is to improve the capabilities of existing bounding surface plasticity models, as this framework is characterised by its modularity and flexibility. As a result, specific components of the formulation of this type of model may be adjusted to improve the reproduction of any aspect of soil behaviour deemed essential to the problem being analysed. In this paper, a series of computational studies are performed in order to establish the impact of expanding a bounding surface plasticity model for sands on its modelling capabilities and to suggest ways of mitigating the associated increase in complexity. Changes to three distinct aspects of the selected constitutive model are examined: the shape of the Critical State Line in $p^{\prime}-e$ space, the expression used for calculating the hardening modulus and the form of the yield surface. It is shown that the introduced changes have the potential to increase significantly the ability to control how certain aspects of soil response, such as degradation of stiffness and flow liquefaction with limited deformation, are reproduced by the model. Moreover, this paper presents a systematic approach to the expansion of this type of constitutive model, establishing 
how alterations to the formulation of a model may be assessed in terms of improved accuracy and potential benefits.

\section{Introduction}

The adequate simulation of certain complex dynamic phenomena is only possible if the constitutive framework employed in the analysis is capable of reproducing a wide range of features of soil behaviour under cyclic loading. One of the strategies proposed to deal with this challenging aspect of numerical modelling is to employ bounding surface plasticity models. Initially developed for metal plasticity [1], this framework uses the distance from the current stress point to its projection on the bounding surface to quantify the material's plastic response. Following early applications of this approach to soil behaviour (e.g. [2]), this type of model was extended by Manzari and Dafalias [3] to incorporate concepts of Critical State Soil Mechanics [4], rendering it a very powerful analysis tool. Given its flexibility and modularity, it is perhaps unsurprising that this model has been constantly extended by other authors in order to increase its capabilities [5-10]. However, as different researchers focus on improving the accuracy of the model when reproducing distinct aspects of soil response, the choice of version depends greatly on the boundary value problem being analysed. In the present case, the formulation proposed by Papadimitriou and Bouckovalas [5] is chosen as a starting point for a detailed computational study on the expansion of this type of model, as it was the first version specifically developed to address the simulation of cyclic loading under a wide range of strain amplitudes. Indeed, such an approach is adopted in order to characterise the effect of altering different components of the model and, therefore, to establish whether the increase in its complexity (e.g. larger number of parameters, higher nonlinearity, etc.) is offset by the associated benefits in terms of added flexibility and accuracy. In this paper, after briefly introducing the original formulation of the model proposed by 
Papadimitriou and Bouckovalas [5], exhaustive studies on the impact of modifying three components of this model are presented: the shape of the Critical State Line $(C S L)$ in $p^{\prime}-e$ space, the expression adopted for the hardening modulus and the form of the yield function.

\section{Original formulation}

As mentioned in the previous section, the chosen starting point for the work presented in this paper is the bounding surface plasticity model described by Papadimitriou and Bouckovalas [5], which is a generalisation to multiaxial space of the model proposed by Papadimitriou et al. [11], as both its elastic and plastic components were specifically designed with the objective of simulating a wide range of features of cyclic soil behaviour. This model, the formulation of which is summarised in Table 1, is an evolution of that proposed by Manzari and Dafalias [3]. Therefore, it includes the same four distinct surfaces in triaxial stress space, each characterised by a constant value of the stress ratio $\eta=q / p^{\prime}$, to define the response of the material (Figure 1 ): the yield surface, the critical state surface, the dilatancy surface and the bounding surface. The first of these surfaces, which determines the onset of plasticity and is defined by Eq. 10, has the shape of a narrow cone with its apex at the origin of the stress space. The intersection of this surface with the deviatoric plane normalised with respect to the mean effective stress, $p^{\prime}$, is a circle of radius proportional to parameter $m$ and centre given by tensor $\boldsymbol{\alpha}$ (Figure 2). Unlike in the model proposed by Manzari and Dafalias [3], this surface only hardens kinematically, meaning that $m$ is constant, while $\alpha$ evolves during shearing. The critical state surface (Eq. 6) defines the failure of the material and its shape in stress space is that of an open wedge (Figure 1) characterised by parameters $M_{c}^{c}$ and $M_{e}^{c}$, which denote the stress ratios at critical state in compression and extension, respectively. Given that for sands these quantities are different, the intersection of this surface with the deviatoric plane, described by Eq. 9a to Eq. 9c is generally noncircular, as 
illustrated in Figure 2. The loading direction, represented in the aforementioned expressions by the Lode's angle, $\theta$, can be evaluated using:

$$
\cos \left(3 \cdot \theta+\frac{\pi}{2}\right)=\frac{3 \cdot \sqrt{3}}{2} \cdot \frac{\overline{J_{3}}}{\left(\overline{J_{2}}\right)^{3 / 2}}
$$

where $\overline{J_{2}}=1 / 2 \cdot \overline{\mathbf{r}}: \overline{\mathbf{r}}, \overline{J_{3}}=1 / 3 \cdot \overline{\mathbf{r}}: \overline{\mathbf{r}}: \overline{\mathbf{r}}$ and $\overline{\mathbf{r}}$ is the radial tensor. The latter is defined as $\overline{\mathbf{r}}=\mathbf{r}-\boldsymbol{\alpha}$, where $\mathbf{r}$ is the normalised deviatoric stress tensor given by $\mathbf{r}=\mathbf{s} / p^{\prime}=\left(\boldsymbol{\sigma}-p^{\prime} \mathbf{I}_{3}\right) / p^{\prime}$. According to Eq. $35, \theta=-\pi / 6$ corresponds to triaxial compression states, while for triaxial extension a value of $\theta=\pi / 6$ is obtained. The dilatancy and bounding surfaces have a shape which is identical to that of the critical state surface (Figure 2), with their openings in stress space being defined by the respective stress ratios under triaxial compression conditions, $M_{c}^{d}$ and $M_{c}^{b}$, defined in Eq. 7 and Eq. 8, respectively. Note that it is assumed that for triaxial extension loading the openings of the two surfaces are determined by $M_{e}^{d}=c \cdot M_{c}^{d}$ and $M_{e}^{b}=c \cdot M_{c}^{b}$, where $c=M_{e}^{c} / M_{c}^{c}$ is a shape factor. Unlike the critical state surface, which is entirely described by the two model parameters $M_{c}^{c}$ and $M_{e}^{c}$, the openings of the dilatancy and bounding surfaces change during shearing, in accordance with Eq. 7 and Eq. 8 introduced by Manzari and Dafalias [3], with the state parameter, $\psi$, proposed by Been and Jefferies [12]:

$$
\psi=e-e_{C S}
$$

where $e$ is the void ratio of the material at a given state and $e_{C S}$ is the void ratio at the $C S L$ corresponding to the effective stress level to which the material is subjected. Similar to several other models based on the same framework (e.g. $[3,10])$, Papadimitriou and Bouckovalas [5] proposed the use of a linear expression for the $C S L$ in the $\ln p^{\prime}-e$ plane, as described by Eq. 5. In effect, the adoption of a relation between the state parameter and the openings of the dilatancy and bounding surfaces (Eq. 7 and Eq. 8) is intended to enable the reproduction of different patterns of mechanical response which have been observed depending on whether the current state is denser-than-critical (i.e. $\psi<0$ ) or looser-than-critical (i.e. $\psi>0$ ). As a result, this 
approach allows the simulation of the behaviour of the material under a wide range of initial states, characterised in terms of mean effective stress and void ratio, using a single set of parameters. However, it then becomes necessary to introduce in the formulation the distances between the current stress point and the different surfaces when determining the plastic behaviour of the material, a procedure for which the definition of a mapping rule is required. In the case of the model presented by Papadimitriou and Bouckovalas [5], the adopted mapping rule, which was originally proposed in [3], is schematically represented in Figure 2 (note that others are possible, as highlighted by Andrianopoulos et al. [13]). Based on this procedure, the projections of the current stress point on the dilatancy and bounding surfaces, denoted as $\boldsymbol{\alpha}^{\mathbf{d}}$ and $\boldsymbol{\alpha}^{\mathbf{b}}$, respectively, may be established using:

$$
\boldsymbol{\alpha}^{\mathbf{d}, \mathbf{b}}=\sqrt{2 / 3} \cdot\left(g(\theta, \mathrm{c}) \cdot M_{c}^{d, b}-m\right) \cdot \mathbf{n}
$$

where $\mathbf{n}$ is the traceless unit tensor obtained by normalising the radial tensor $\overline{\mathbf{r}}$ by its dimension:

$$
\mathbf{n}=\frac{\overline{\mathbf{r}}}{\sqrt{2 / 3} \cdot m}
$$

Given the above definitions, the distances between the current stress point and its respective image points, expressed for convenience in terms of the back stress tensor rather than stress tensor, can be calculated by:

$$
d^{d, b}=\left(\boldsymbol{\alpha}^{\mathbf{d}, \mathbf{b}}-\boldsymbol{\alpha}\right): \mathbf{n}
$$

The distance to the dilatancy surface, $d^{d}$, is used to calculate the volumetric component of the plastic potential (Eq. 12), with a positive value of $d^{d}$ (i.e. the stress point is within the surface) implying plastic contraction of the material, while a negative value of $d^{d}$, which occurs when the stress point is outside the surface, leads to plastic dilation. Note that by comparing the gradients of the yield surface and plastic potential, $\mathbf{\partial F} / \partial \boldsymbol{\sigma}$ (Eq. 11a) and $\mathbf{\partial P} / \partial \boldsymbol{\partial}$ (Eq. 12), respectively, it can be concluded that, in general, this constitutive model does not employ an associated flow rule. Conversely, the distance to the bounding surface, $d^{b}$, affects directly the magnitude and sign of 
the hardening modulus, $A$ (Eq. 13a), meaning that a positive value of $d^{b}$ leads to the simulation of the hardening of the material, while softening occurs when $d^{b}$, and therefore $A$, are negative. Indeed, the latter aspect of the model distinguishes it from other bounding surface plasticity models (e.g. $[14,15])$, as it implies that it is possible for the stress point to be located outside the bounding surface.

In addition to the position of the stress point in relation to the bounding surface, the value of the hardening modulus is also affected by the value of $h_{f}$, which is a scalar computed based on the evolution of the fabric tensor (Eq. 13d). This aspect of the formulation, which was firstly proposed by Papadimitriou et al. [11] and then further developed for inclusion in the version of the model described by Papadimitriou and Bouckovalas [5], is defined by Eq. 15a to Eq. 15d. Its introduction improved considerably the simulation of soil response under undrained cyclic loading and, as a result, it has been retained by subsequent versions of this constitutive model (e.g. [10]). It should be noted, however, that the evolution of the fabric tensor and its contribution to the plastic response of the material only take place for samples which are initially in a denser-than-critical state (i.e. $\psi_{0}<0$ in Eq. 15c).

Lastly, Papadimitriou and Bouckovalas [5] proposed a cyclic non-linear formulation based on a Ramberg-Osgood approach [16] for the elastic component of the model (Eq. 1 to Eq.4). This addition successfully improved the simulation of cyclic soil response at small-strains, both in terms of stiffness degradation and hysteretic damping, resulting in its adoption by subsequent models based on the same framework (e.g. $[9,10])$. It essentially consists of the adoption of the expression proposed by Hardin and Richart [17], which establishes the maximum shear modulus of the material based on the current mean effective stress level and void ratio (Eq. 1), coupled with a degradation law (Eq. 2a) based on the quantity:

$$
\chi_{\text {ref }}^{r}=\sqrt{1 / 2 \cdot\left(\mathbf{r}-\mathbf{r}^{\mathbf{S R}}\right):\left(\mathbf{r}-\mathbf{r}^{\mathbf{S R}}\right)} \quad \text { Eq. } 40
$$


which corresponds to the distance measured in the deviatoric plane between the current stress state, $\mathbf{r}$, and that at the last shear reversal, $\mathbf{r}^{\mathbf{S R}}$. The latter, together with $p^{\prime S R}, G_{\max }^{S R}$ in Eq. $2 \mathrm{~b}$, are updated whenever a shear reversal takes place, a situation which is deemed to have occurred when a given incremental solicitation reduces the value of the scalar $\chi_{r e f}^{e}$ defined as:

$$
\chi_{\text {ref }}^{e}=\sqrt{1 / 2 \cdot\left(\mathbf{e}-\mathbf{e}^{\mathbf{S R}}\right):\left(\mathbf{e}-\mathbf{e}^{\mathbf{S R}}\right)} \quad \text { Eq. } 41
$$

where $\mathbf{e}=\boldsymbol{\varepsilon}-\varepsilon_{v o l} / 3 \cdot \mathbf{I}_{3}$ is the deviatoric strain tensor, $\boldsymbol{\varepsilon}$ is the strain tensor and $\varepsilon_{v o l}=\varepsilon_{x}+$ $\varepsilon_{y}+\varepsilon_{z}$ is the volumetric strain. Clearly, by comparing Eq. 40 and Eq. 41, it can be observed that $\chi_{r e f}^{e}$ represents the equivalent distance to $\chi_{r e f}^{r}$ but in strain space. Moreover, the value of scalar $N$ in Eq. 2a, which is initially set to 1 , is altered to 2 when the first shear reversal is detected. This procedure scales the stress-strain curve by a factor of 2 , thus ensuring that the simulated cyclic response complies with the original Masing rules $([18,19])$. To complete the definition of the elastic stiffness of the material, a constant Poisson's ratio, $v$, is assumed (Eq. 4).

The model proposed by Papadimitriou and Bouckovalas [5] clearly presents considerable advancements over the original formulation by Manzari and Dafalias [3], particularly with respect to the simulation of soil behaviour under cyclic loading. Furthermore, the modularity and flexibility of the framework upon which it is based allow for alterations to its initial formulation to be gradually introduced and thoroughly tested. This process, which is described in the following sections, was carried out by firstly implementing the model proposed by Papadimitriou and Bouckovalas [5] into the Finite Element code ICFEP [20], a procedure described by Taborda [21]. Subsequently, three distinct components of the model, namely the shape of the CSL in $e-p^{\prime}$ space, the expression used in the calculation of the hardening modulus, $A$, and the yield surface, were independently modified and the respective impact on the simulation of soil behaviour was characterised. In each of the discussed tests, unless otherwise stated, the parameters for Nevada sand proposed by Papadimitriou and Bouckovalas [5] were used (Table 2). 


\section{Shape of the Critical State Line in $p^{\prime}-e$ space}

The first aspect of the model to be investigated was the shape of the $C S L$ in the $e-p^{\prime}$ space, as it controls the value of the state parameter, $\psi$, a concept which occupies a central role in its formulation. As described in the previous section, Papadimitriou and Bouckovalas [5] proposed the use of a linear expression in $\ln p^{\prime}-e$ space (Eq. 5), in accordance with the principles of critical state soil mechanics established for clays [4]. However, results obtained from undrained triaxial compression tests, such as those shown in Figure 3a for Leighton Buzzard sand presented by Been et al. [22], show that a single linear expression is unable to describe the observed $C S L$ for the complete range of stress values. As a result, a hypothetical stress path under undrained conditions starting from a void ratio of 0.80 and a mean effective stress of $100 \mathrm{kPa}$ (point $A$ in Figure 3a), would reach critical state at drastically different stress levels (points $B$ and $C$ ), depending on which of the linear approximations for the $C S L$ would be adopted.

The first solution to improve the reproduction of the observed CSL for a wide range of values of stress consisted of using a bilinear form, such as the one depicted in Figure 3b [22]. However, while this approach addressed the main concerns raised over the adoption of a single linear expression, the use of a logarithm function still had the major drawback of predicting high void ratios at critical state for very low stresses. Indeed, it was observed by Verdugo and Ishihara [23] that the CSL seems to curve towards a horizontal position as the stress level decreases, intercepting the axis of zero mean effective stress at a value which can be considered to be close to the maximum void ratio at atmospheric pressure [24]. As a result, to reproduce the required shape at both low and high values of mean effective stress, a power law was proposed by Li and Wang [25]: 


$$
e_{C S}=\left(e_{C S}\right)_{r e f}-\lambda \cdot\left(\frac{p^{\prime}}{p_{\text {ref }}^{\prime}}\right)^{\xi}
$$

where $\xi$ is an additional parameter controlling the overall curvature of the $C S L$, while $\left(e_{C S}\right)_{r e f}$, rather than being related to the reference pressure $p_{\text {ref }}^{\prime}$, is now the void ratio under zero mean effective stress. The advantages of using such a form are clearly illustrated in Figure $3 \mathrm{~b}$, where a unique set of parameters $-\left(e_{C S}\right)_{\text {ref }}=1.01 ; \lambda=0.08$ and $\xi=0.35$ - is capable of adequately reproducing the entire collection of laboratory results. Therefore, given its flexibility and accuracy, it is unsurprising that most versions of the presented model have adopted a power law for the $\operatorname{CSL}$ (e.g. $[6,8,9,26,27])$. However, it should be noted that the quality of the approximation provided by the power law appears to degrade for extremely high stress levels (i.e. $p^{\prime}>10 M P a$ ), a range where, if deemed necessary, a linear form seems to be more appropriate [24].

To characterise the impact of altering the expression used for the $C S L$ on the modelled soil response, a series of undrained triaxial compression tests on Nevada sand was simulated. The two adopted CSLS were those proposed by Papadimitriou and Bouckovalas [5] for the linear shape and by Ling and Yang [28] for the power law. The corresponding parameters are listed in Table 3 and the resulting CSLs are depicted in Figure 4. In all tests, an initial mean effective stress of $45 \mathrm{kPa}$ was chosen, since the two CSLs coincided at this point, thus guaranteeing similar values of the state parameter at the start of shearing. Furthermore, the analyses were conducted for two distinct values of density, $e_{1}=0.80$ and $e_{2}=0.70$, in order to investigate the effect of the distance to the $C S L$ on the differences obtained in soil behaviour. The four distinct tests are labelled as 1.L, 1.P, 2.L and 2.P, where the number refers to the void ratio of the material and the letter to the shape of the $C S L$, with $\mathrm{L}$ corresponding to the linear expression and $\mathrm{P}$ to the power law.

The results for the looser samples (1.L and 1.P) are presented in terms of stress-strain curve and generation of excess pore water pressures in Figure $5 a$ and $5 b$, respectively. From Figure 4 , it is 
evident that an undrained test starting from position 1 would first meet the linear CSL. As a consequence, the axial strain at which critical state was reached was slightly lower when this form, rather than the power law, was used. Since no other significant differences can be detected, it can be concluded that, for this initial state and test type, the adopted shape of $C S L$ does not appear to be important. In Figure $6 a$ and $6 b$, the results for the denser samples (2.L and 2.P) are shown in terms of stress-strain response and evolution of excess pore water pressures, respectively. Clearly, unlike for the tests on looser samples, there is a great disparity in the axial strain levels required to reach critical state: $3 \%$ when the power law was adopted and $40 \%$ when the linear form was used. Similarly, the stress state and excess pore water pressure predicted at this stage were also extremely different, with the test where a power law was adopted (2.P) terminating at $p^{\prime}=1000$ $\mathrm{kPa}$ and $\Delta u=-500 \mathrm{kPa}$, while the one using a linear expression (2.L) carried on to reach $p^{\prime}=16000$ $\mathrm{kPa}$ and $\Delta u=-8000 \mathrm{kPa}$. Naturally, these are very significant discrepancies, illustrating that the choice of the shape of $C S L$ does have a great influence on the modelled soil behaviour. In particular, it may be concluded that the adoption of a linear expression is not recommended as it may lead to the severe overestimation of the maximum stress and strain levels that a relatively dense material is able to sustain prior to reaching failure. This limitation has obviously more grave implications when analysing the performance of structures transmitting large loads to the soil, such as footings and piles.

\section{Formulation of the hardening modulus}

\subsection{General considerations}

By comparing the various evolutions of the model originally proposed by Manzari and Dafalias [3], it is easily concluded that the hardening modulus is the component which registers the largest number of different formulations. Despite the evident diversity, the expressions can be essentially described as a product of a value $h$ by the mean effective stress, $p^{\prime}$, and the distance between the 
current stress point and the bounding surface, $d^{b}$. As described by Eq. 13a, Papadimitriou and Bouckovalas [5] proposed that the value of $h$ should be calculated based on the current distance to the bounding surface in relation to its overall diameter, an aspect summarised by $h_{b}$ (Eq. $13 \mathrm{~b}$ and Eq. 13c), and the current characteristics of the fabric tensor, $\mathbf{F}$, which is introduced in the hardening modulus by the scalar $h_{f}$ (Eq. 13d). Despite the already substantial sophistication of this expression, it is important to observe that it does not take into account important aspects such as the density and elastic stiffness of the material. Moreover, the function defining the influence of the distance from the current stress point to the bounding surface may be altered in order to enable the degradation rate of the plastic modulus with the shearing process to be adjusted. However, independently of the extent of algebraic changes needed to solve these perceived limitations of the model, it is important to allow the possibility of reverting to the original expression by choosing an appropriate set of parameters. This latter aspect is particularly important in cases where the calibration procedure indicates that the influence of any of the variables involved is not sufficiently significant to be considered. Clearly, this observation extends to the influence of the mean effective stress, which is already a part of the model through the adopted expression for $h_{b}$ (Eq. 13b).

It should be noted that unlike the modifications introduced to the shape of the $C S L$, which are supported by results from laboratory tests, altering the hardening modulus lacks a well-defined physical motivation. Consequently, the impact of the changes described in the following sections on the simulated soil behaviour will only be illustrated through parametric studies. Moreover, the parameters proposed for Nevada sand by Papadimitriou and Bouckovalas [5] (Table 2) will be used, with the exception of $H_{0}$, which will be set to 0.0 in order to remove the effect of fabric on the magnitude of the hardening modulus.

\subsection{Effect of void ratio}


The influence of the density of the material on the hardening modulus can be introduced by replacing the constant $h_{0}$, which is a material parameter in the original formulation (Eq. 13b), by the linear function of the void ratio, $h_{e}$ (Eq. 43). The resulting form is similar to that found in other versions of the model (e.g. [6]) and requires the definition of only one additional parameter $(\gamma)$. However, since for very loose samples the proposed expression may lead to the undesirable situation where negative values of $h_{e}$ are calculated, a cut-off level for the void ratio, $e_{\max }$, corresponding to a minimum value of $h_{e}$ (Eq. 44), is necessary (alternatively, one could have adopted a power law [9]).

$$
\begin{array}{ll}
h_{e}=h_{0} \cdot(1-\gamma \cdot e) \geq h_{e, \min } & \text { Eq. } 43 \\
h_{e, \min }=h_{0} \cdot\left(1-\gamma \cdot e_{\max }\right) & \text { Eq. } 44
\end{array}
$$

To illustrate the impact of the proposed change to the formulation of the hardening modulus, undrained triaxial compression tests were simulated for two distinct situations: one where a constant value of $h_{e}$ was used, as enforced by the original formulation of the model $\left(h_{0}=5000.0\right.$ and $\gamma=0.0$ ), and one using a variable $h_{e}$, defined by $h_{0}=16666.7$ and $\gamma=1.0$. Note that the magnitude of $\gamma$ was chosen according to data found in the literature for similar sands [6], while the corresponding value of $h_{0}$ was determined by imposing that both sets of parameters should yield the same value $\left(h_{e}=5000.0\right)$ for a void ratio of 0.70 . Moreover, since $\gamma$ was introduced with the intent of improving the accuracy of the model for a wider range of soil densities without requiring any adjustment of parameters, the true impact of adopting a linear function for $h_{e}$ can only be illustrated if simulations for at least two different initial void ratios are compared. However, to allow for clear conclusions to be drawn, the average $h_{e}$ for the two simulations should be identical to the value used with the original formulation (i.e $h_{e}=5000.0$ ). In the present case, the void ratios of $0.65\left(h_{e}=5833.3\right)$ and $0.75\left(h_{e}=4166.7\right)$ were chosen. All the tests started from an isotropic stress state of $p^{\prime}=100 \mathrm{kPa}$ and the results obtained are shown in Figure 7. 
As expected, the results of the simulations confirmed that the global stiffness of the looser sample ( $e=0.75)$ decreased when a variable $h_{e}$ was employed $(\gamma=1.0)$, while for the denser sample $(e=$ 0.65) a stiffer response was observed. Furthermore, it is important to note that, if one would assume that real soil behaviour would coincide with that obtained for $\gamma=1.0$, then the use of a constant value of $h_{e}=5000.0$ would have resulted in a relatively poor approximation. Thus, the performed parametric study shows that the introduction of the extra parameter does increase the flexibility of the model, improving its accuracy when the modelling of materials with significantly different densities is required. Similarly, the modified formulation of the hardening modulus may have a substantial impact when dealing with sands which undergo densification, for example during post-liquefaction reconsolidation. However, if no sufficient experimental evidence is available or if it is concluded that an average value of $h_{e}$ provides sufficient precision, then the original formulation can be employed by simply setting $\gamma$ to 0.0 .

\subsection{Relationship with the elastic stiffness}

The Ramberg-Osgood formulation was originally proposed for the elastic component of the constitutive model by Papadimitriou et al. [11] and was retained by Papadimitriou and Bouckovalas [5] as a form of improving the simulation of the variation of stiffness over a wide range of deformation levels. However, given that the size of the yield surface is usually small, plasticity tends to dominate the modelled response for the majority of the shearing process. Therefore, since the latter type of behaviour is generally controlled by the flow rule and the hardening modulus, the adoption of a Ramberg-Osgood approach may be insufficient to guarantee that the stiffness of the material degrades in a consistent way as shearing progresses. To illustrate such a situation, three undrained triaxial compressions tests on samples consolidated isotropically to $p^{\prime}=100 \mathrm{kPa}$ with a void ratio of 0.70 were simulated using distinct values of parameter $\gamma_{1}$ : $2.5 \times 10^{-4}$, which is the original value proposed by Papadimitriou and Bouckovalas [5] for Nevada 
sand (Table 2), $2.5 \times 10^{-3}$ and $2.5 \times 10^{-2}$. The obtained results, in terms of stress-strain curves and variation of secant shear stiffness with strain level, are illustrated in Figure 8.

By increasing the magnitude of parameter $\gamma_{1}$, which controls the strain level above which no degradation of the elastic shear modulus takes place, it would be expected that an increasingly stiffer response would be observed. However, as it can be seen in Figure 8, for axial strain levels between $0.05 \%$ and $0.35 \%$, the abovementioned trend is inverted, with the simulation using the highest value of $\gamma_{1}$ being characterised by the lowest shear stiffness. Thus, to guarantee that the specified degradation of the elastic stiffness is adequately reflected on the plastic stiffness of the material, it is proposed to include the current value of the elastic shear modulus as an additional factor in the expression of the hardening modulus (Eq. 45). Clearly, by setting parameter $\alpha$ to 0.0 , the original formulation of the model is obtained. Moreover, it should be noted that although a specific law is not usually defined, other versions of the model presented in the literature also include the elastic shear modulus in the determination of the plastic modulus (e.g. $[6,8,9])$, which is achieved by introducing the additional multiplier $h_{g}$ :

$$
h_{g}=G_{\text {tan }}^{\alpha}
$$

The previously described simulations were repeated for $\alpha=1$, meaning that a full contribution of the elastic shear stiffness to the value of the hardening modulus was assumed. Moreover, with the inclusion of $h_{g}$, the magnitude of $h_{0}$ was reduced from 5000.0 to 0.1450 to ensure that the global value of the hardening modulus at the start of shearing would be similar to that employed in the previous set of tests. Clearly, as it can be seen in Figure 9a, the relative positions of the stressstrain curves for the whole range of axial strain levels are in accordance with the relation between the values of $\gamma_{1}$ employed in each situation, with larger values of this parameter leading to stiffer responses, as confirmed by the variations of secant shear stiffness with deformation level (Figure 9b). Therefore, the introduction of the nonlinear stiffness in the calculation of the hardening 
modulus establishes an efficient link between elastic and plastic behaviour, yielding more consistent results.

\subsection{Distance to the bounding surface}

The last of the modifications introduced to the formulation of the hardening modulus concerns the function which defines the contribution of the distance between the current stress point and the bounding surface, $d^{b}$. In the expression presented by Papadimitriou and Bouckovalas [5], which was inherited from the early Manzari and Dafalias [3] proposal, the effect of the proximity to the bounding surface is given by the factor $m_{b}$ :

$$
m_{b}=\frac{\left|d^{b}\right|}{\left\langle d_{r e f}^{b}-\left|d^{b}\right|\right\rangle}=\frac{\frac{\left|d^{b}\right|}{d_{r e f}^{b}}}{\left\langle 1-\frac{\left|d^{b}\right|}{d_{r e f}^{b}}\right\rangle}
$$

where $d_{r e f}^{b}$ is the opening of the bounding surface measured along the current loading direction (Eq. $13 \mathrm{c}$ in Table 1) and \langle\rangle are the Macauley brackets (i.e. $\langle x\rangle=x$ if $x>0$ and $\langle x\rangle=0$ if $x<0$ ). As a result, Eq. 46 returns positive values ranging from 0 (stress point is on the bounding surface, $d^{b}=0$ ) to infinity (point on the opposite extremity of the bounding surface, $d^{b}=d_{r e f}^{b}$ ). However, these are not the true limiting cases since, as it has been referred to before, the stress point can be located outside the bounding surface, meaning that two additional situations can occur: $d^{b}<0$ (point above the bounding surface) and $d^{b} \geq d_{r e f}^{b}$ (point on or below the opposite extremity of the bounding surface). Indeed, it was perhaps to prevent unreasonable values from being obtained when calculating $m_{b}$ for the first of these two conditions, that the modulus operator was included in Eq. 46 by [3] . Conversely, the use of the Macauley brackets, proposed by [11] and retained by [5], implies that an infinite value of the plastic modulus should be calculated for the second of the aforementioned cases, a potentially problematic situation from a computational point of view, which requires appropriate care when implementing the model in a finite element 
code. The expression for $m_{b}$ (Eq. 46) was later altered by Li and Dafalias [26] with one of the arguments being that an infinite hardening modulus should be predicted at the beginning of the shearing process, in order to ensure a smooth transition from elastic to plastic behaviour. In fact, subsequent versions of the model generally adopted this new proposal (e.g. [6]) or a variation of it (e.g. [9]). Other recent versions (e.g. [8]), however, abandoned this approach and reverted to a form similar to the original Manzari and Dafalias [3] expression, amply demonstrating the lack of consensus over the formulation of this component of the model.

A complete assessment and comparison between the different approaches would necessarily involve the need to implement both and carry out an exhaustive testing programme. Since a clear conclusion about this issue cannot be found in the literature, there is no apparent motivation to change the type of function chosen by Papadimitriou and Bouckovalas [5]. It is however acknowledged, that an identical procedure to that suggested by Loukidis and Salgado [9] could increase the flexibility of the model and could be, therefore, beneficial to its overall performance. Eq. 47 shows the altered expression, where $\beta$ is a new material parameter. Note that, similar to the other proposed alterations, its value can be set to 0.0 if the original formulation is to be used.

$$
m_{b}=\left(\frac{\left|d^{b}\right|}{\left\langle d_{r e f}^{b}-\left|d^{b}\right|\right\rangle}\right)^{\beta+1}
$$

Figure 10 shows the effect of $\beta$ on the values returned by the new function. As it can be seen, increasing the value of $\beta$ leads to a faster decrease of the function in the regions closer to the bounding surface $\left(d^{b} / d_{r e f}^{b}<0.5\right)$ and to higher values being used for points located further away from this surface. To illustrate the impact of this modification on the modelled soil behaviour, undrained triaxial compression tests on samples isotropically consolidated to $p^{\prime}=100 \mathrm{kPa}$ and with a void ratio of 0.70 were simulated for $\beta=0.0$ (original formulation), $0.25,0.50$ and 1.00 .

In the first set of tests, which focussed on the impact on the modelled response during the initial stages of shearing, $h_{0}$ was recalculated for each value of $\beta$ in order to match the stress-strain 
curve registered for axial strains above $3 \%$ when employing the original formulation (i.e. $\beta=0.0$ and $\left.h_{0}=5000.0\right)$. The obtained results are shown in Figure 11 . Clearly, by varying parameter $\beta$, it is possible to control certain aspects of the stress path in $p^{\prime}-q$ space, such as the stress state at which phase transformation, i.e. the quasi-steady state as defined by Ishihara [29], occurs. In fact, as $\beta$ increased, the minimum mean effective stress registered during the test also increased significantly. Moreover, the shape of the stress-strain curves suggests that $\beta$ also influences the variation of stiffness during the subsequent dilatant stage. For the second parametric study, $h_{0}$ was recalculated for each value of $\beta$ in order for the stress state at phase transformation to be identical in all the tests. As the resulting stress paths and stress-strain curves depicted in Figure 12 illustrate, parameter $\beta$ influences the stress state at which the temporary peak in deviatoric stress is reached, before dropping towards the quasi-steady state. In fact, for larger magnitudes of $\beta$, a higher peak value is obtained and larger deformations are needed before the dilatant phase is initiated. The possibility of controlling the characteristics of flow liquefaction with limited deformation, which is an important feature of sand behaviour [29], reinforces the usefulness of introducing the proposed changes to the formulation of the hardening modulus.

\section{Low-stress yield surface}

The framework upon which the presented model is based relies greatly on the position of the stress state in the deviatoric plane to determine the behaviour of the material. In fact, the normalisation of the stress tensor by the mean effective stress is a recurring aspect of the formulation. This particularity of the model naturally raises concerns about its precision in situations where the stress level is very low, such as when liquefaction is being simulated. Thus, to avoid potential losses of accuracy and to reduce the computational cost of the integration of the constitutive equations under these conditions, an additional yield surface, termed "secondary", is proposed. The chosen form is simple and consists of imposing a limit value to the mean effective 
stress, $p_{Y S}^{\prime}$, as defined in Eq. 48. Note that the subscript " 2 " is introduced in order to distinguish the equations presented in this section from those used for the original yield surface.

$$
F_{2}=p_{Y S}^{\prime}-p^{\prime}=0
$$

The plastic behaviour predicted when this yield surface is activated is characterised by an associated flow rule (Eq. 49) and is considered to be perfectly plastic. Thus, for this surface, $p_{Y S}^{\prime}$ is a material constant, rather than a hardening parameter, and the corresponding hardening modulus, $A_{2}$, is $0.0[20]$.

$$
\frac{\partial F_{2}}{\partial \sigma^{\prime}}=\frac{\partial P_{2}}{\partial \sigma^{\prime}}=\frac{1}{3} I_{3}
$$

Based on these expressions, the calculation of the resulting incremental plastic strains, $\Delta \boldsymbol{\varepsilon}_{\mathbf{2}}^{\mathbf{p}}$, follows the procedure outlined in Appendix A, allowing for changes in hardening parameters to be determined. Indeed, although the fabric tensor, F, has not been considered when describing the plastic behaviour introduced by this surface, both its spherical and deviatoric components must still be updated (Eq. 50 and Eq. 51). The other hardening parameter, the tensor $\boldsymbol{\alpha}$, remains unchanged as it is exclusively used to characterise the position of the original yield surface. In the event of both primary and secondary yield surfaces being activated, additional modifications are needed to determine the incremental plastic strains and to formulate the elasto-plastic matrix, as described in Appendix B.

$$
\begin{gathered}
\Delta f_{p}=H \cdot \Delta \varepsilon_{v o l, 2}^{p} \\
\Delta \mathbf{f}=-H \cdot\left\langle-\Delta \varepsilon_{v o l, 2}^{p}\right\rangle \cdot[C \cdot \mathbf{n}+\mathbf{f}]
\end{gathered}
$$

The precise magnitude of parameter $p_{Y S}^{\prime}$, which determines the position of the newly introduced yield surface, is difficult to estimate. It is expected, however, that a small number will not efficiently solve issues related to loss of precision due to low values of mean effective stress, while large values will alter significantly the behaviour predicted by the model. Consequently, tests must be conducted to evaluate the impact of $p_{Y S}^{\prime}$. In the present case, a cyclic undrained direct shear 
test on Nevada sand, identical to that used by Papadimitriou and Bouckovalas [5] to demonstrate the capabilities of the original constitutive model, was used. The initial conditions are characterised by $\sigma_{v}^{\prime}=160.0 \mathrm{kPa}, \tau_{x y}=5.9 \mathrm{kPa}$ and an initial void ratio of 0.66 , while the applied load amplitude was $13.7 \mathrm{kPa}$ (i.e. shear stresses vary between $-7.8 \mathrm{kPa}$ and $19.6 \mathrm{kPa}$ ). Three different positions of this surface were tested by setting parameter $p_{Y S}^{\prime}$ to $0.1 \mathrm{kPa}, 1.0 \mathrm{kPa}$ and $10.0 \mathrm{kPa}$

The results obtained in the simulations are shown in Figure 13 in terms of evolution of excess pore water pressures and shear strain with the number of cycles. Clearly, with respect to the former aspect of cyclic soil behaviour, the effect of the new yield surface is restricted to the maximum value registered, which, as expected, decreased for larger values of $p_{Y S}^{\prime}$. Conversely, the shear strain histories registered important differences during the final loading cycles. Perhaps the most evident impact of the activation of the secondary yield surface was obtained for $p_{Y S}^{\prime}=10.0 \mathrm{kPa}$, where the computed deformations were much lower than those observed in the remaining analyses. By imposing a minimum magnitude to the mean effective stress, a lower limit on the value of the hardening modulus linked to the primary yield surface was also introduced. As a result, the almost perfectly plastic response (i.e. $A_{1} \approx 0.0$ ) exhibited under low stresses was substituted by a more controlled deformation pattern.

The two remaining analyses also yielded rather different results. In particular, a sharp decrease in the shear strain while unloading from a shear stress of $19.6 \mathrm{kPa}$ to $-7.8 \mathrm{kPa}$ was only noticeable for $p_{Y S}^{\prime}$ of $1.0 \mathrm{kPa}$. Subsequently, upon reversal of the loading direction, most of this accumulated shear strain was recovered, leading to levels comparable to those registered when $p_{Y S}^{\prime}$ is $0.1 \mathrm{kPa}$. This effect was caused by the interaction between the secondary yield surface and the evolution of the fabric tensor. Indeed, for the mean effective stress to remain constant, the incremental elastic volumetric strains were required to be zero. Therefore, for undrained conditions, the incremental plastic volumetric strain must also be zero, thus impeding any changes in the 
spherical and deviatoric components of the fabric tensor (Eq. 15a and 15b, respectively). As a result, when the loading direction changes, the magnitude and, consequently, the effect of this tensor on the hardening modulus is different.

In conclusion, the introduction of the proposed secondary yield surface has been shown to successfully prevent the mean effective stress from reaching values below the limit defined by $p_{Y S}^{\prime}$. Clearly, the use of large values for this parameter, as demonstrated by the results of the presented parametric study, may restrict the ability of the model to simulate liquefaction-related phenomena, namely the full development of excess pore water pressures under cyclic loading and the large shear strains observed under such conditions. Conversely, only modest differences were registered between the results for $p_{Y S}^{\prime}=0.1 \mathrm{kPa}$ and $1.0 \mathrm{kPa}$, suggesting that using a relatively low value for this parameter benefits the stability of the model without substantially compromising its capabilities. Therefore, as a general guideline, the position of the secondary yield surface and its impact on the simulated soil response should be carefully investigated both during model calibration and the analysis of boundary value problems.

\section{Performance of the modified constitutive model}

The introduction of alterations to the formulation of the constitutive model was presented in the previous sections from an abstract perspective, focussing mainly on characterising to which extent different aspects of soil response were affected by each of the proposed modifications. Indeed, the performed studies have shown that the modified model is inherently more flexible than the original proposal upon which it is based, though its complete definition now requires the determination of six additional parameters (see Table 4).

To demonstrate its modelling capabilities, the modified version of the model was calibrated based on the results of the extensive laboratory testing on Nevada sand carried out for the VELACS 
project [30,31]. The adopted procedure, based on similar techniques as those proposed in [11] and [9], is described in detail by Taborda [21] and led to the parameters listed in Table 5.

Given that one of the potential advantages of basing the formulation of the constitutive model on the concept of state parameter is to be able to simulate sand behaviour under a wide range of initial conditions, a set of five undrained triaxial compression tests performed on samples of Nevada sand prepared with distinct values of relative density and consolidated to different mean effective stress levels were chosen to assess the performance of the model. The first set of comparisons is illustrated in Figure 14 and concerns the effect of initial mean effective stress (40 $\mathrm{kPa}, 80 \mathrm{kPa}$ and $160 \mathrm{kPa}$ ) on the behaviour of samples at relative density of $60 \%$. Conversely, the impact of varying density $(40 \%, 60 \%$ and $70 \%)$ on the mechanical response of samples isotropically consolidated to a value of mean effective stress of $80 \mathrm{kPa}$ is depicted in Figure 15, together with the corresponding numerical predictions. As it can be seen, in both cases, the constitutive model is capable of accurately reproducing the observed stress-strain behaviour and the excess pore water pressure generated during shearing.

In terms of undrained cyclic behaviour of Nevada sand, both a direct simple shear test and a triaxial test performed on this material were simulated. Note that, similar to the simulated monotonic triaxial tests, cyclic tests characterised by different combinations of densities and initial stress levels were selected: $D_{r}=45.9 \%$ and $\sigma_{v, 0}^{\prime}=80 \mathrm{kPa}$ for the direct simple shear test and $D_{r}=$ $62.0 \%$ and $p_{0}^{\prime}=40 \mathrm{kPa}$ for the triaxial test. The numerical predictions are illustrated in Figure 16, where it can be seen that the model is capable of reproducing the observed mechanical response for both loading modes and initial conditions.

\section{Conclusions}


The flexibility and modularity of the constitutive model described by Papadimitriou and Bouckovalas [5], which is an evolution of the model originally proposed by Manzari and Dafalias [3] and a generalisation of the model presented in Papadimitriou et al. [11], were explored through a series of computational studies. Three distinct components of the model were identified as needing improvement - the shape of the $C S L$ in $p^{\prime}-e$ space, the expression of the hardening modulus and the yield surface - and the effect of changing their formulation on the simulated soil behaviour was characterised.

Specifically, it was shown that the adoption of a power law for describing the $C S L$, which is amply supported by experimental evidence, greatly affects the prediction of the deformation level required for the material to reach Critical State, in particular for very dense sands. Subsequently, three distinct alterations to the hardening modulus were independently introduced and their respective impact on soil response analysed: the dependency of this quantity on the void ratio of the material, its link to the elastic stiffness and the nonlinearity of the function defining the influence of the proximity of the current stress state to the bounding surface. With respect to the first of these changes, the performed parametric study demonstrated that the new formulation has the potential to extend, if required, the accuracy of the model to a wider range of material densities without the need to perform any adjustment to the parameters used. Naturally, this capability is important when dealing with situations where a substantial variation of void ratio exists, either in space (i.e. deposits composed of both loose and dense sand layers) or in time (i.e. repeated liquefaction and solidification of sand deposits leading to an increase in its density). In a second set of analysis, it was shown that the inclusion of the elastic shear modulus in the expression of the hardening modulus resulted in a more consistent behaviour being obtained. Similarly, by introducing a new parameter defining the nonlinearity of the relationship between the hardening modulus and the proximity to the bounding surface, the ability of the model to simulate flow liquefaction with limited deformation, an important feature of undrained soil 
response, became controllable. Lastly, a new low-stress yield surface was introduced in the model formulation. The performed parametric study illustrated that, unless its position is adequately chosen, the use of the new yield surface may lead to the simulation of unrealistic behaviour. However, unlike the previous modifications, this change was motivated by the need to limit the observed detrimental effect on the computation time of the highly non-linear behaviour simulated by the model under low mean effective stress levels.

The modified formulation of the model is described in Table 4, where it can be seen that six new parameters are now required $\left(\xi, \alpha, \beta, \gamma, e_{\max }\right.$ and $\left.p_{Y S}\right)$. It is important to note that, with the exception of the shape of the $C S L$, all changes can be deactivated by setting the associated parameters to 0.0 , thus guaranteeing compatibility with the formulation originally proposed by Papadimitriou and Bouckovalas [5]. Moreover, this allows any of the new features to be removed whenever there is lack of experimental evidence supporting its use (e.g. no observed effect of the void ratio on the hardening modulus). Consequently, rather than focusing solely on expanding the original model, this paper presents a systematic approach to characterise the benefits of adjusting specific components of its formulation and whether they are offset by the increased complexity. Lastly, the modified formulation of the constitutive model was shown to be capable of accurately reproducing the undrained behaviour of Nevada sand under monotonic and cyclic loading for a wide range of initial relative densities and mean effective stress levels.

\section{Acknowledgements}

The authors would like to thank FCT - Fundação para a Ciência e Tecnologia, Portugal, for sponsoring the doctoral programme of D.M.G. Taborda at Imperial College London (SFRH/BD/21453/2005), during which this work was developed. 


\section{References}

[1] Dafalias YF, Popov EP. Model of nonlinearly hardening materials for complex loading. Acta Mechanica. 1975;21(3):173-92.

[2] Dafalias YF. Bounding Surface Plasticity .1. Mathematical Foundation and Hypoplasticity. Journal of Engineering Mechanics, ASCE. 1986;112(9):966-87.

[3] Manzari MT, Dafalias YF. A critical state two-surface plasticity model for sands. Geotechnique. 1997;47(2):255-72.

[4] Schofield AN, Wroth CP. Critical state soil mechanics. London: McGraw-Hill, 1968.

[5] Papadimitriou AG, Bouckovalas GD. Plasticity model for sand under small and large cyclic strains: a multiaxial formulation. Soil Dynamics and Earthquake Engineering. 2002;22(3):191-204.

[6] Dafalias YF, Manzari MT. Simple plasticity sand model accounting for fabric change effects. Journal of Engineering Mechanics, ASCE. 2004;130(6):622-34.

[7] Dafalias YF, Papadimitriou AG, Li XS. Sand Plasticity Model Accounting for Inherent Fabric Anisotropy. Journal of Engineering Mechanics. 2004;130(11):1319-33.

[8] Taiebat M, Dafalias YF. SANISAND: Simple anisotropic sand plasticity model. International Journal for Numerical and Analytical Methods in Geomechanics. 2008;32(8):915-48.

[9] Loukidis D, Salgado R. Modeling sand response using two-surface plasticity. Computers and Geotechnics. 2009;36(1-2):166-86.

[10] Andrianopoulos KI, Papadimitriou AG, Bouckovalas GD. Bounding surface plasticity model for the seismic liquefaction analysis of geostructures. Soil Dynamics and Earthquake Engineering. 2010;30(10):895-911.

[11] Papadimitriou AG, Bouckovalas GD, Dafalias YF. Plasticity model for sand under small and large cyclic strains. Journal of Geotechnical and Geoenvironmental Engineering. 2001;127(11):97383.

[12] Been K, Jefferies MG. A state parameter for sands. Geotechnique. 1985;35(2):99-112. 
[13] Andrianopoulos K, Papadimitriou A, Bouckovalas G. Bounding surface models of sands: Pitfalls of mapping rules for cyclic loading. Proceedings, 11 th International Conference on Computer Methods and Advances in Geomechanics. 2005:241-8.

[14] Whittle A, Kavvadas M. Formulation of MIT-E3 Constitutive Model for Overconsolidated Clays. Journal of Geotechnical Engineering. 1994;120(1):173-98.

[15] Grammatikopoulou A, Zdravkovic L, Potts DM. The influence of previous stress history and stress path direction on the surface settlement trough induced by tunnelling. Geotechnique. 2008;58(4):269-81.

[16] Ramberg W, Osgood WR. Description of stress-strain curves by three parameters. National Advisory Committee for Aeronautics, 1943.

[17] Hardin BO, Richart FE, Jr. Elastic wave velocities in granular soils. Journal of the Soil Mechanics and Foundations Division, ASCE. 1963;89(SM1):33-65.

[18] Masing G. Eigenspannungen und Verfestigung beim Messing. 2nd International Conference of Applied Mechanics. Zurich, Switzerland1926.

[19] Kramer SL. Geotechnical Earthquake Engineering. New Jersey: Prentice Hall, 1996.

[20] Potts DM, Zdravković LT. Finite element analysis in geotechnical engineering: theory. London: Thomas Telford, 1999.

[21] Taborda DMG. Development of constitutive models for application in soil dynamics [Ph.D. thesis]. London: Imperial College London, 2011.

[22] Been K, Jefferies MG, Hachey J. The Critical State of Sands. Geotechnique. 1991;41(3):365-81. [23] Verdugo R, Ishihara K. The steady state of sandy soils. Soils and Foundations. 1996;36(2):8191

[24] Klotz EU, Coop MR. On the identification of critical state lines for sands. Geotechnical Testing Journal. 2002;25(3):289-302. 
[25] Li XS, Wang Y. Linear representation of steady-state line for sand. Journal of Geotechnical and Geoenvironmental Engineering. 1998;124(12):1215-7.

[26] Li XS, Dafalias YF. Dilatancy for cohesionless soils. Geotechnique. 2000;50(4):449-60.

[27] Li XS, Dafalias Y, Wang ZL. State-dependant dilatancy in critical-state constitutive modelling of sand. Canadian Geotechnical Journal. 1999;36(4):599-611.

[28] Ling HI, Yang ST. Unified sand model based on the critical state and generalized plasticity. Journal of Engineering Mechanics, ASCE. 2006;132(12):1380-91.

[29] Ishihara K. Soil Behaviour in Earthquake Geotechnics. Oxford: Oxford University Press, 1996.

[30] Arulanandan K, Scott RF. Verification of Numerical Procedures for the Analysis of Soil Liquefaction Problems, Proceedings of the International Conference, 17-20 Oct. 1993, Davis, California. Rotterdam, The Netherlands, Balkema, 1993.

[31] Arulmoli K, Muraleetharan KK, Hossain MM, Fruth LS. VELACS: Verification of liquefaction analyses by centrifuge studies - Laboratory testing program and soil data report. The Earth Technology Corporation, 1992. 


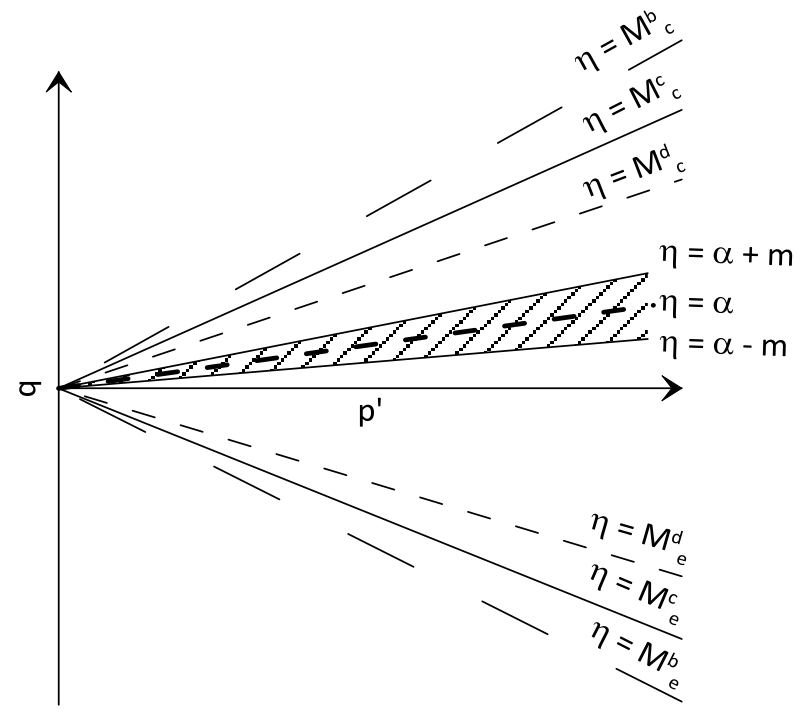

Figure 1: the model surfaces in triaxial stress space. 


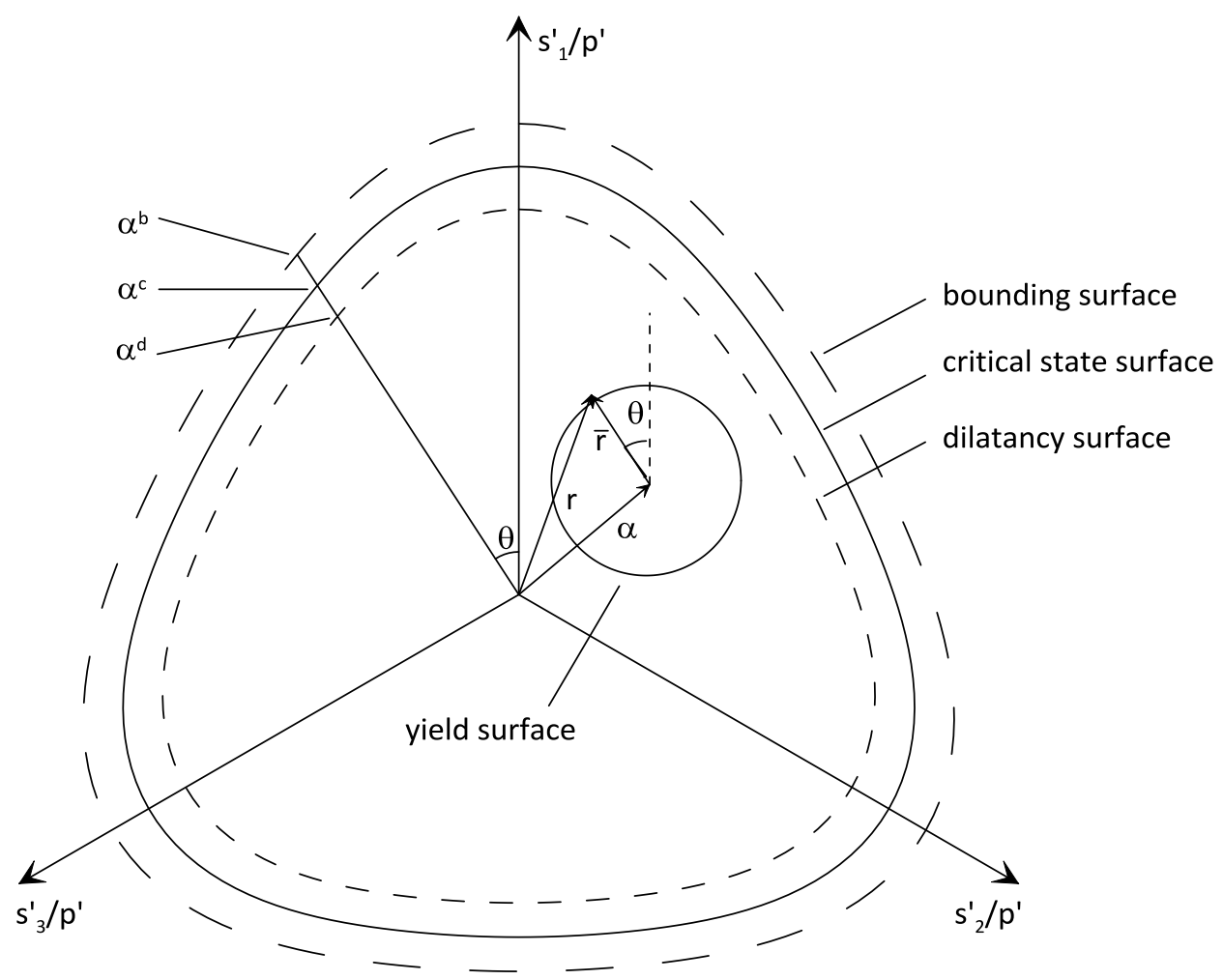

Figure 2: shape of the model surfaces in the deviatoric plane. 


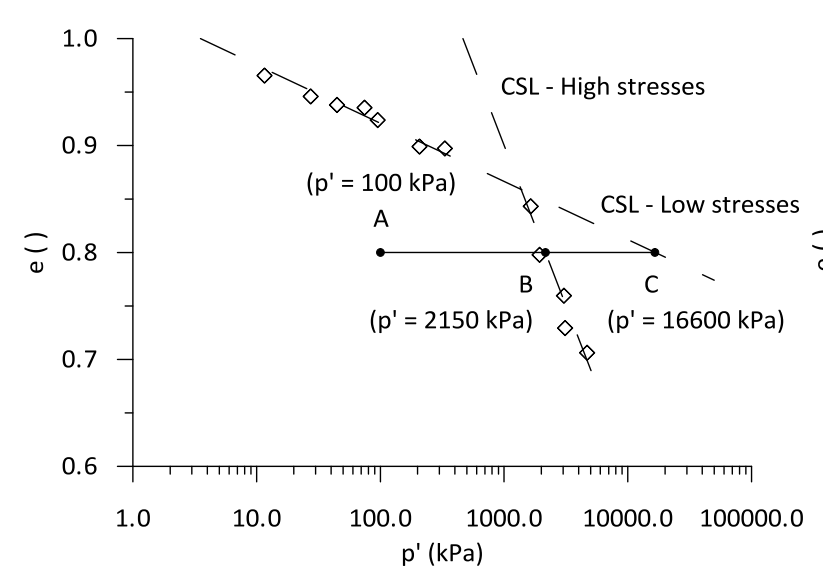

(a)

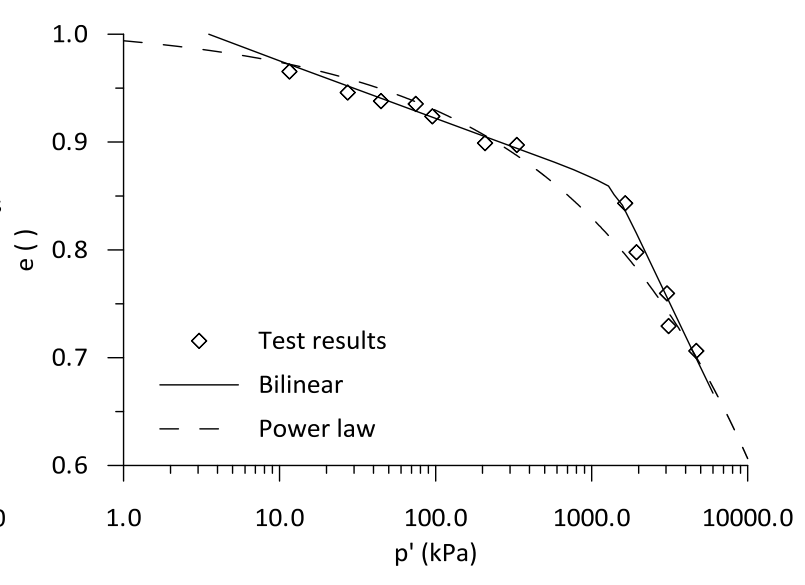

(b)

Figure 3: Location of possible Critical State Lines for Leighton Buzzard sand - (a) results of undrained triaxial compression tests [22] and (b) approximations provided by bilinear and power law expressions. 


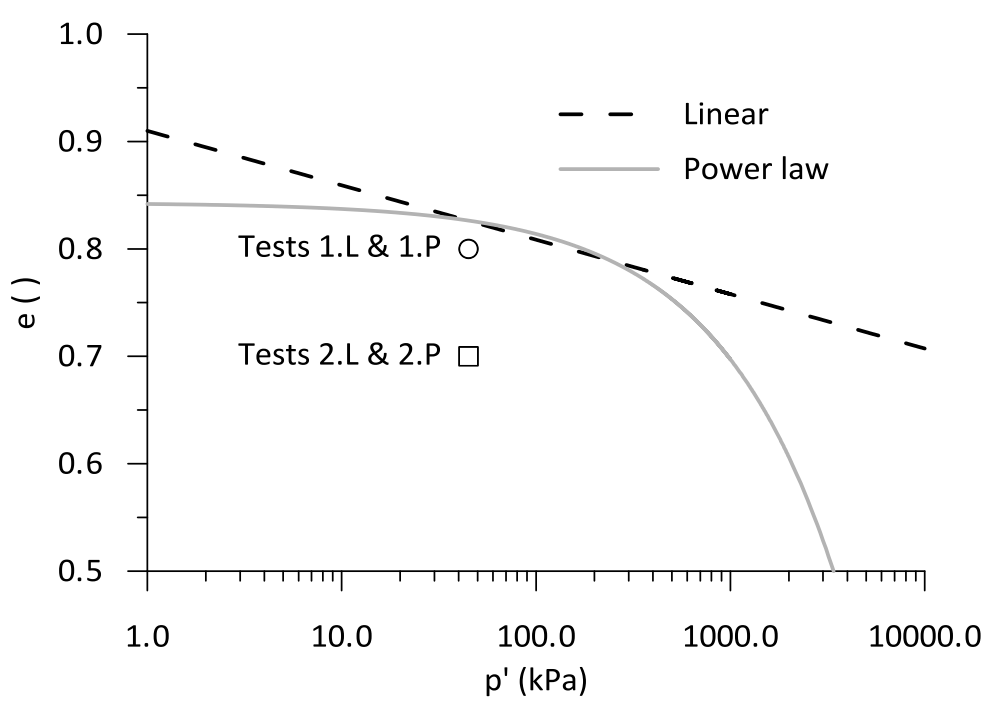

Figure 4: Shape of the Critical State Line and initial conditions of the different tests. 


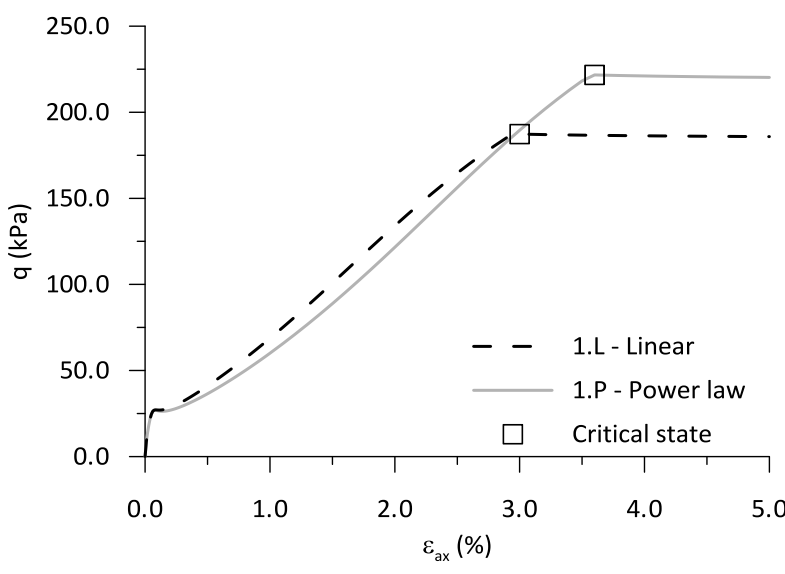

(a)

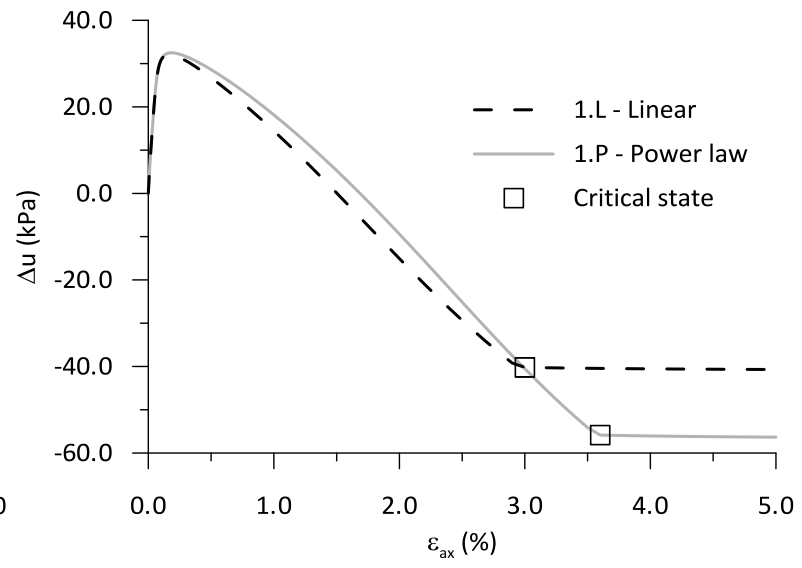

(b)

Figure 5: Results obtained for the lower density material $\left(e_{0}=0.80\right)-(a)$ stress-strain curve and $(b)$ excess pore water pressure. 


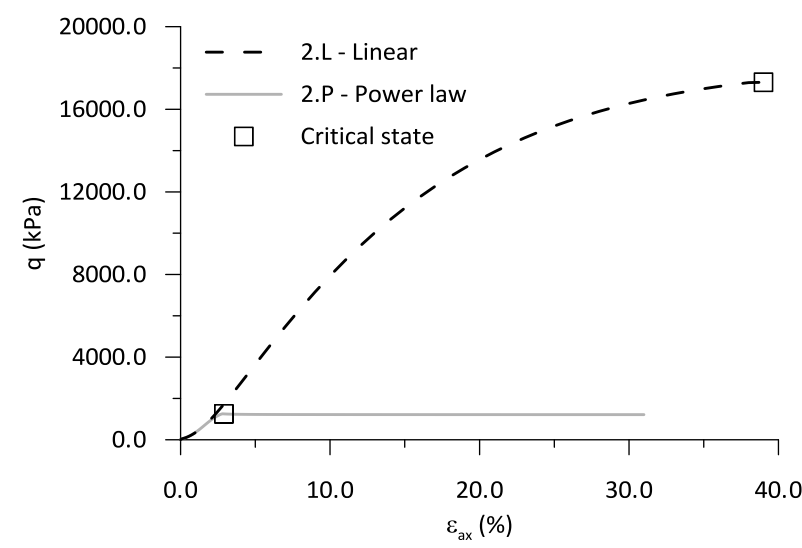

(a)

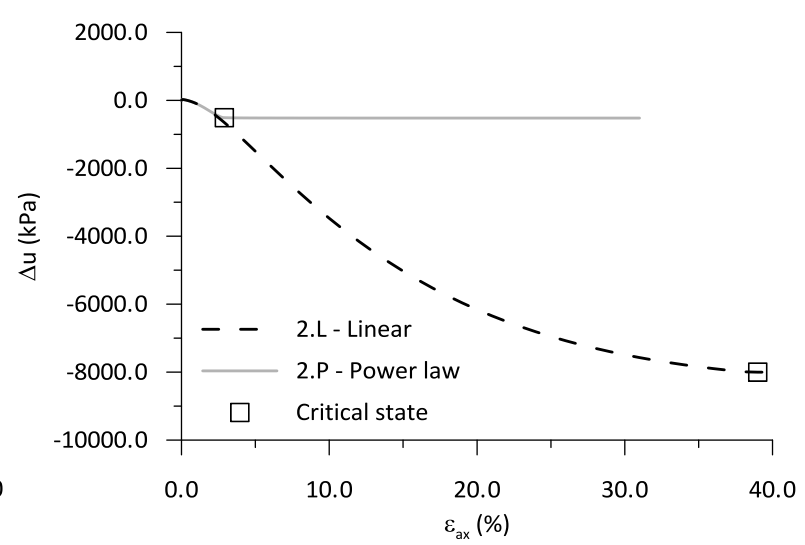

(b)

Figure 6: Results obtained for the higher density material $\left(e_{0}=0.70\right)-(a)$ stress-strain curve and (b) excess pore water pressure. 


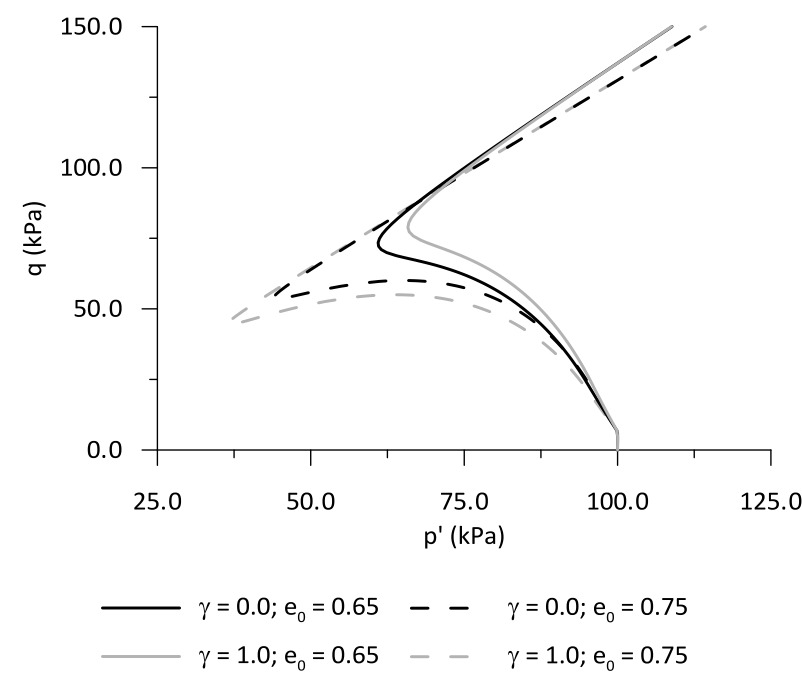

(a)

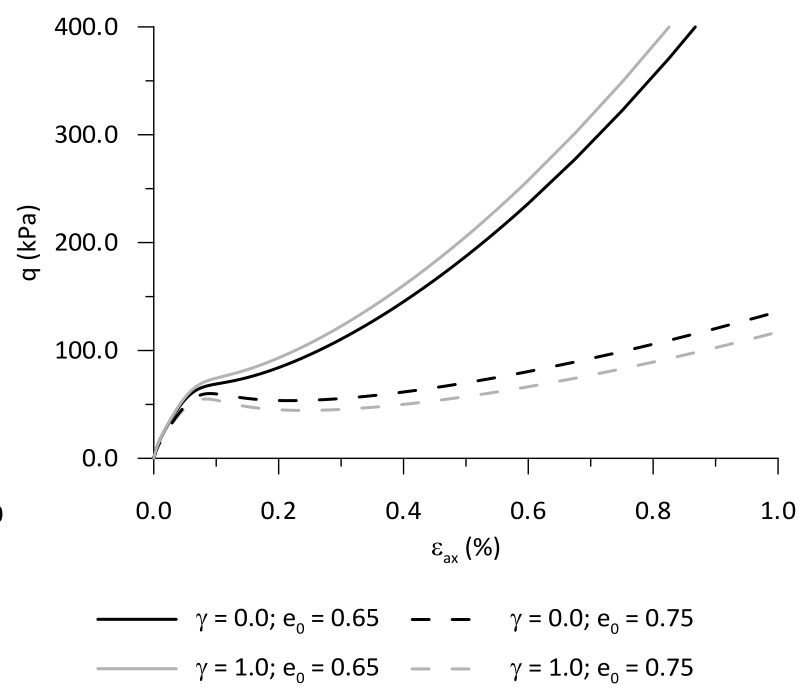

(b)

Figure 7: Influence of the inclusion of void ratio in the hardening modulus - (a) stress path in $p^{\prime}-q$ space and (b) stress-strain curve. 


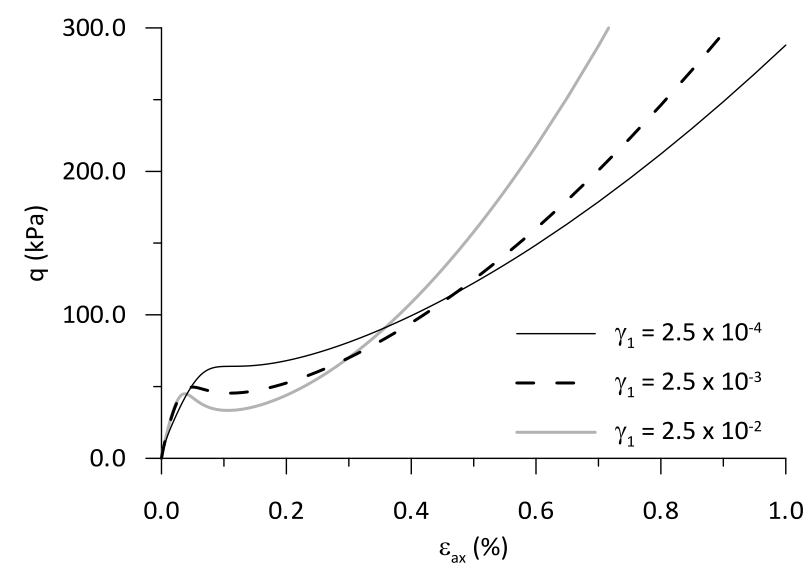

(a)

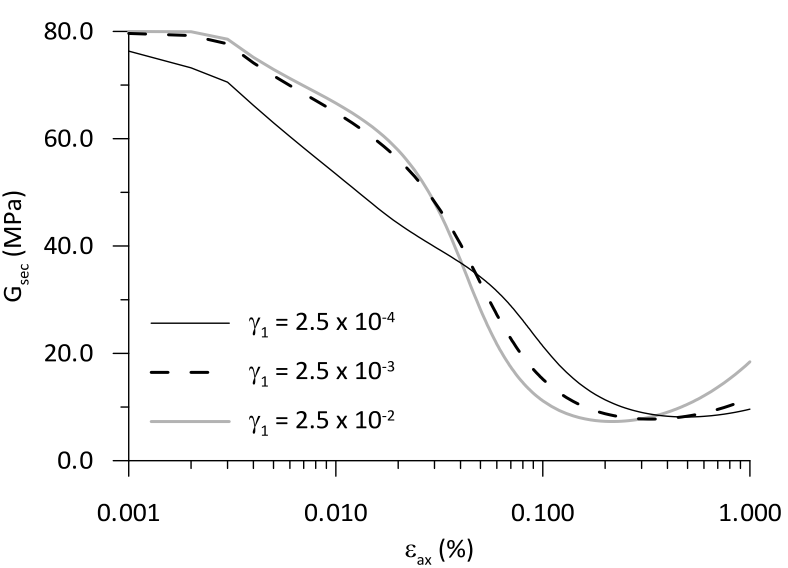

(b)

Figure 8: Influence of the limit strain $\gamma_{1}$ using the original formulation [5] - (a) stress-strain curve and (b) stiffness variation with strain. 


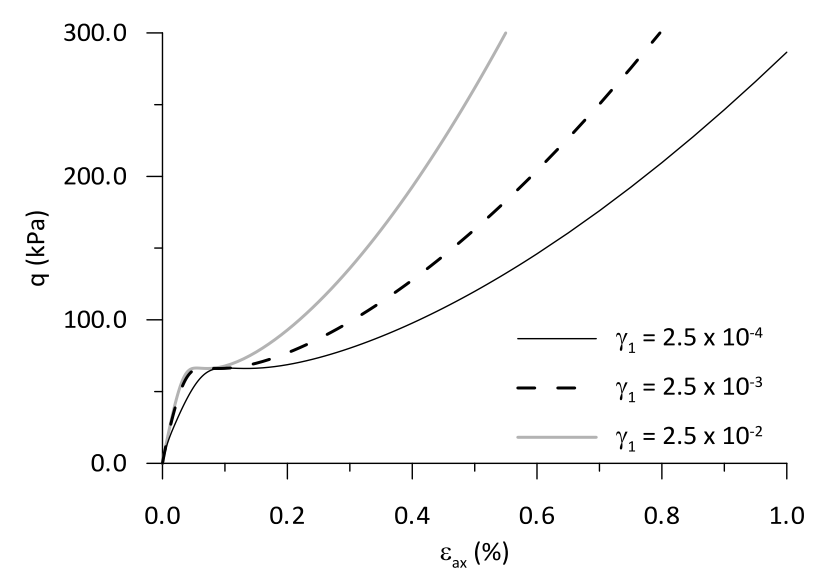

(a)

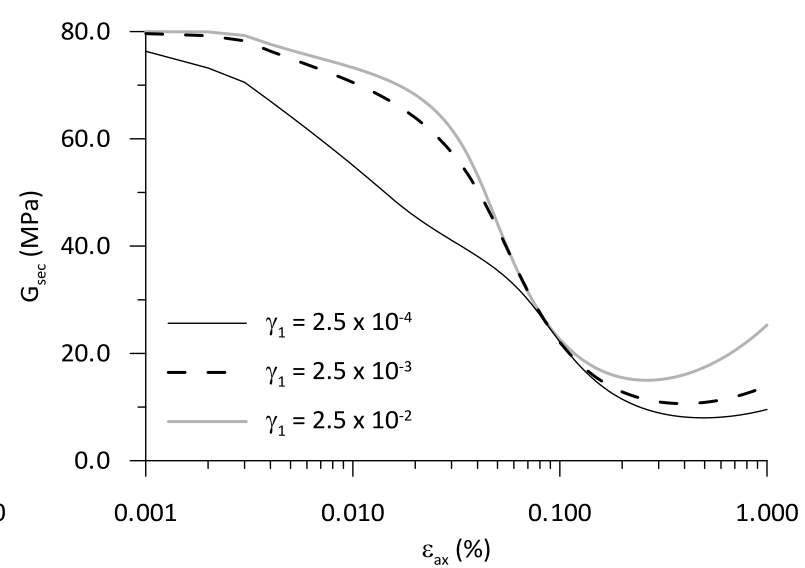

(b)

Figure 9: Influence of the limit strain $\gamma_{1}$ for $\alpha=1-$ (a) stress-strain curve and (b) stiffness variation with strain. 


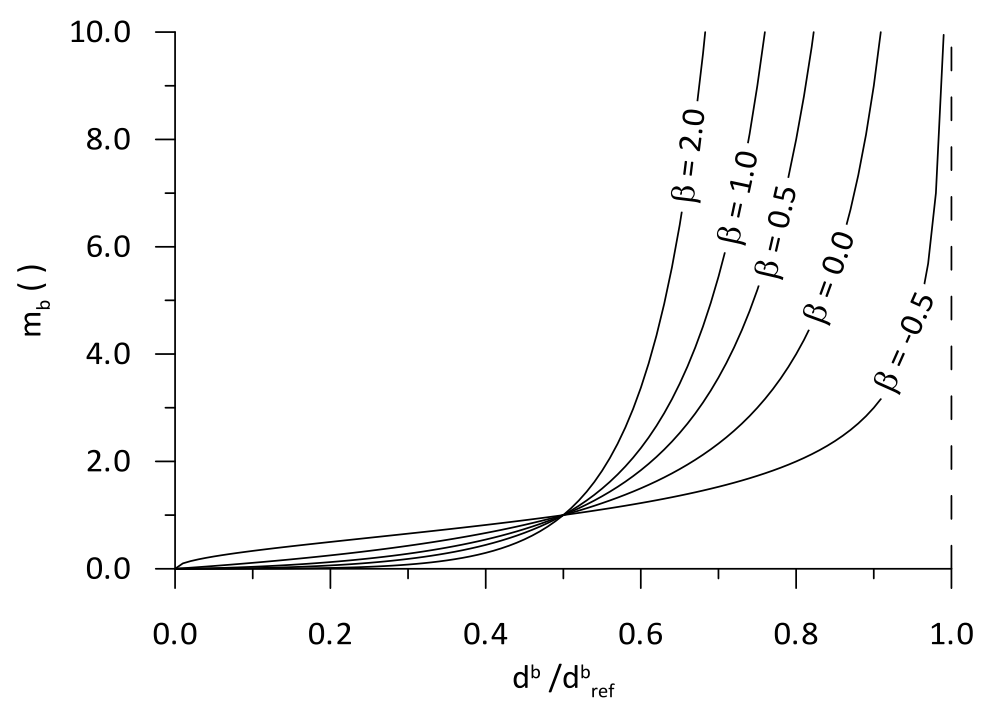

Figure 10: Effect of parameter $\beta$ on the component of the hardening modulus related to the distance to the bounding surface. 


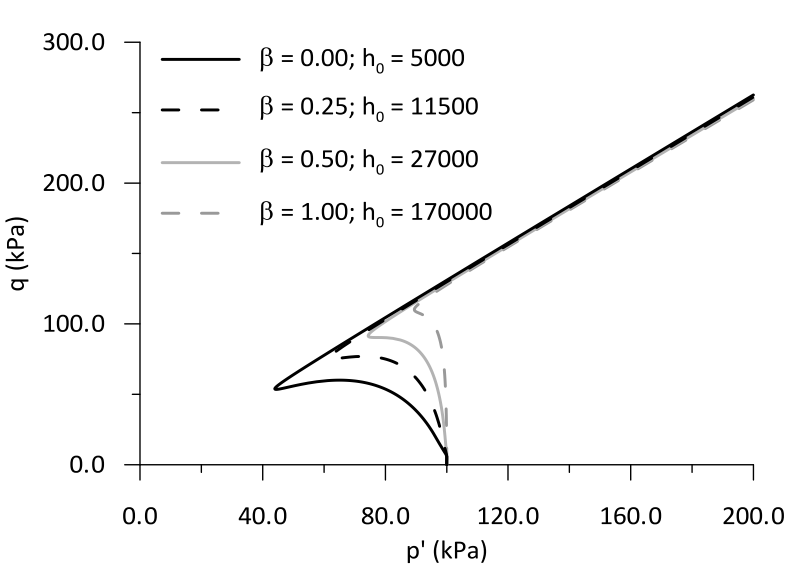

(a)

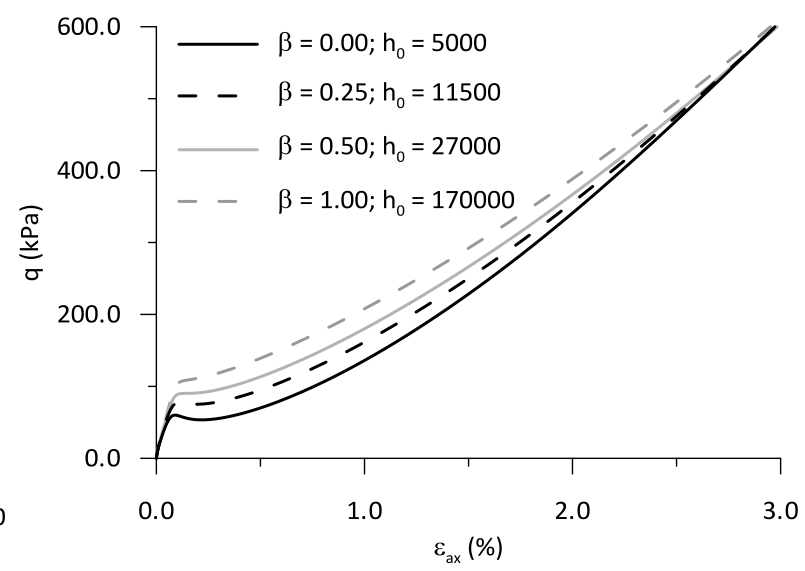

(b)

Figure 11: Influence of parameter $\beta$ for similar global behaviour - (a) stress path in $p^{\prime}-q$ space and stress-strain curve. 


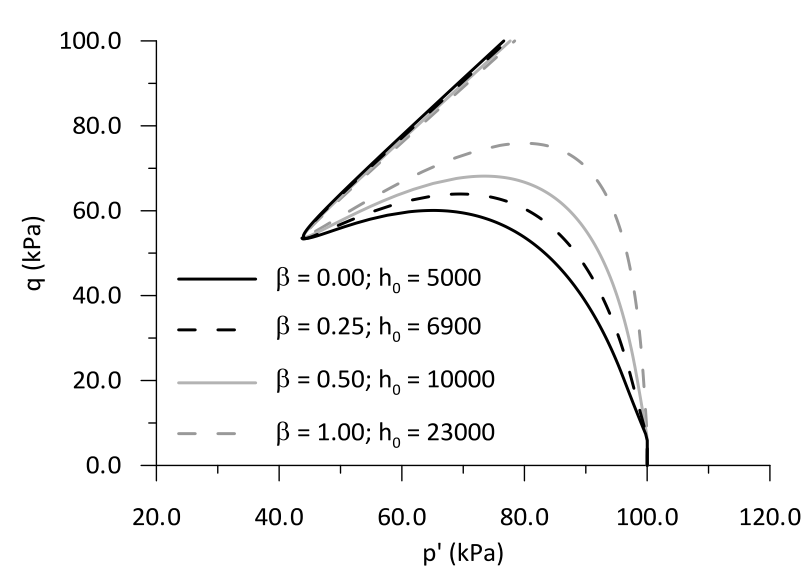

(a)

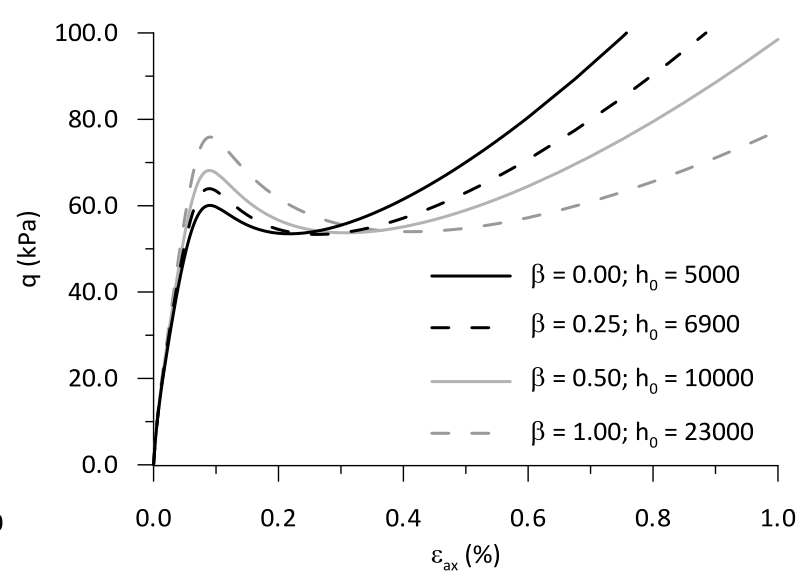

(b)

Figure 12: Influence of parameter $\beta$ for similar minimum $p^{\prime}-\left(\right.$ a) stress path in $p^{\prime}-q$ space and stress-strain curve. 


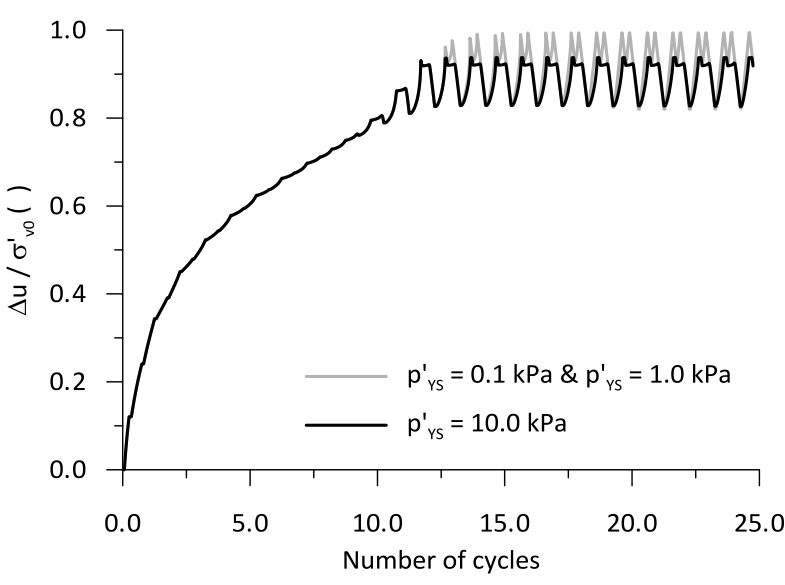

(a)

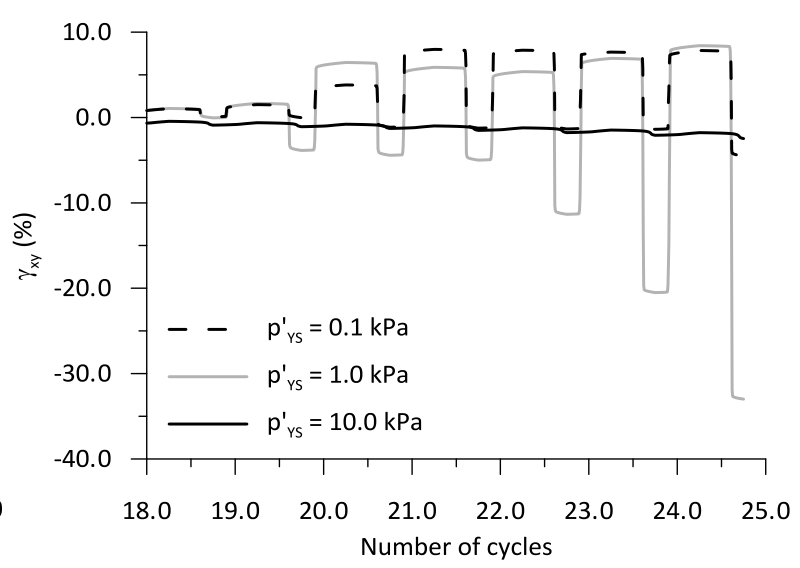

(b)

Figure 13: Influence of the position of the secondary yield surface on the results of the cyclic direct shear test - (a) generation of excess pore water pressures and (b) evolution of shear strain with number of cycles. 


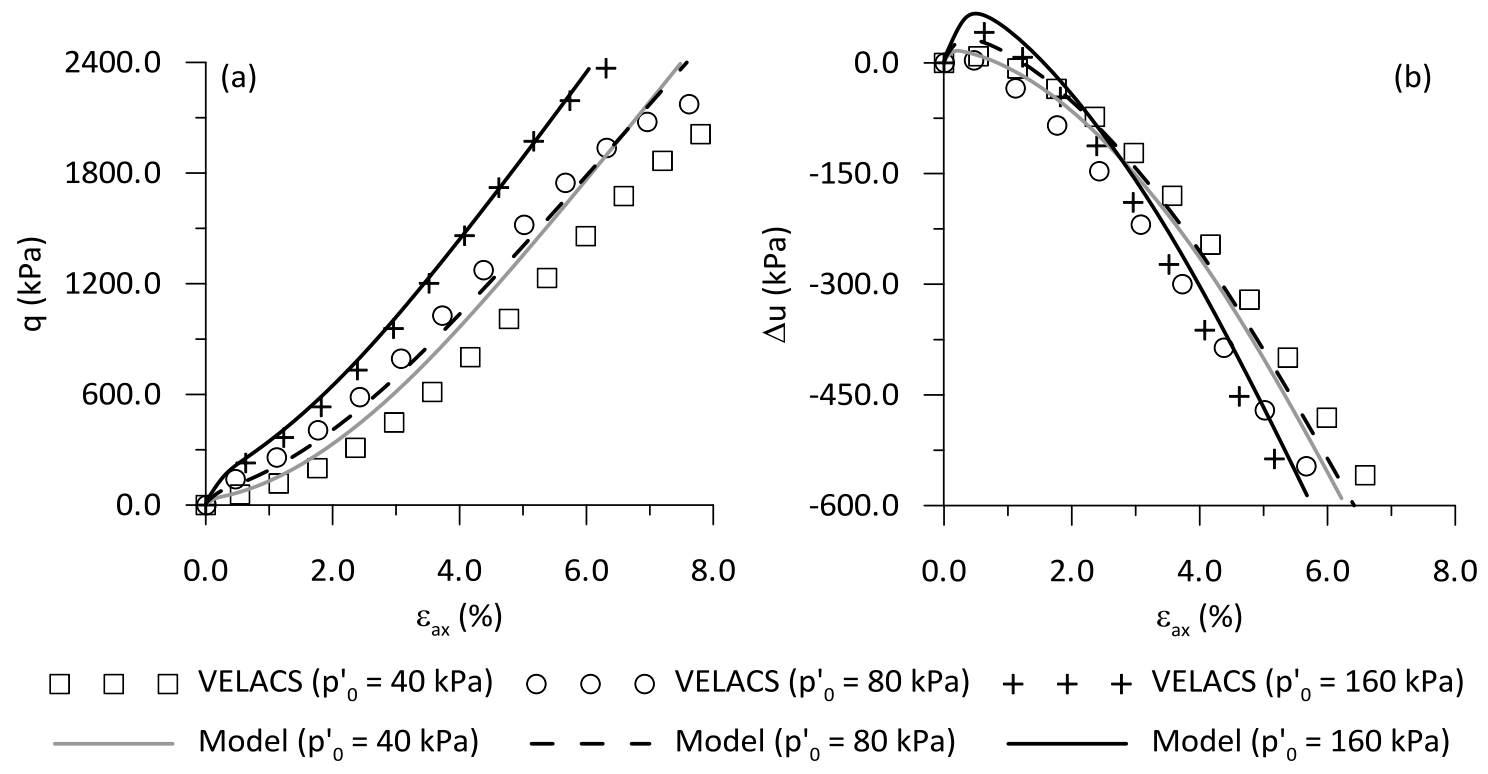

Figure 14: Simulation of undrained triaxial compression tests performed on samples of Nevada Sand [31] with a relative density of $60 \%$ isotropically consolidated under different stress levels (a) stress-strain curve and (b) generation of excess pore water pressures. 

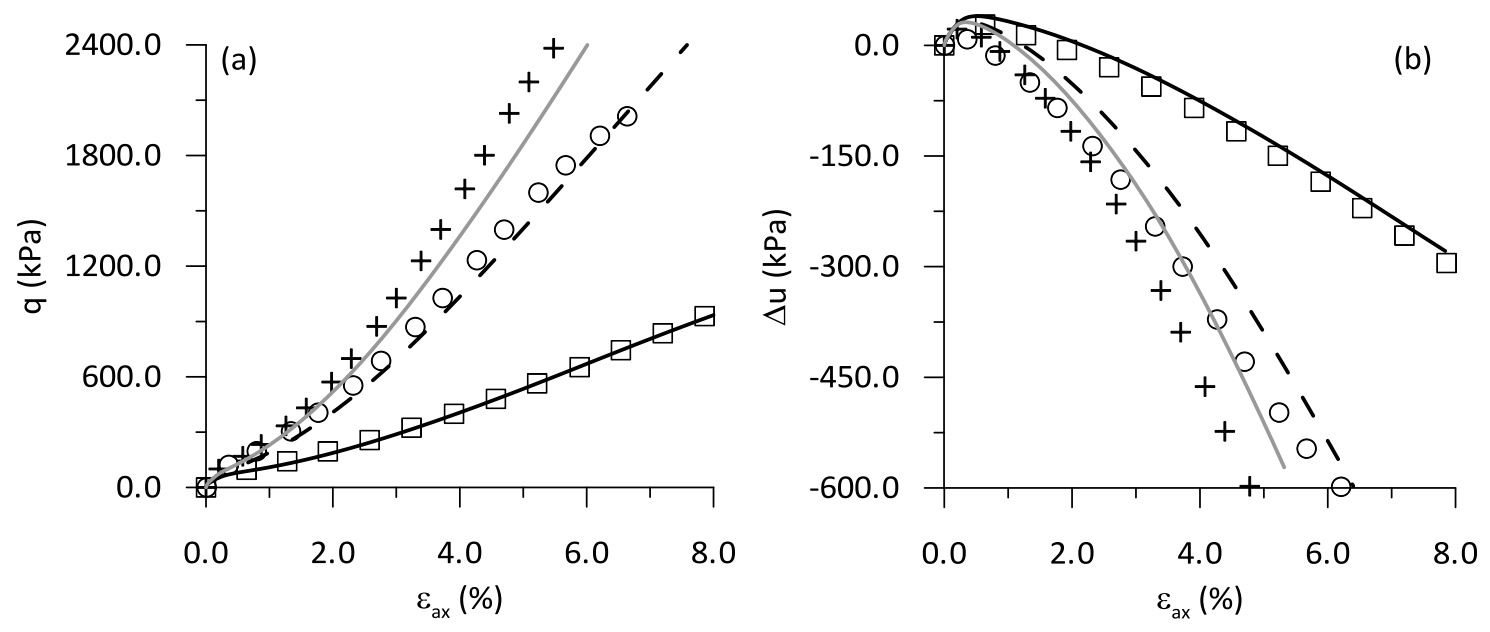

$\square \quad \square \operatorname{VELACS}(\mathrm{Dr}=42.2 \%) \quad \bigcirc \quad \bigcirc \quad \mathrm{VELACS}(\mathrm{Dr}=59.6 \%)+++\operatorname{VELACS}(\mathrm{Dr}=67.1 \%)$

Model $(\mathrm{Dr}=42.2 \%) \quad-\quad-\operatorname{Model}(\mathrm{Dr}=59.6 \%)$ Model $(\mathrm{Dr}=67.1 \%)$

Figure 15: Simulation of undrained triaxial compression tests performed on samples of Nevada Sand [31] of different density isotropically consolidated to a mean effective stress of $80 \mathrm{kPa}-(\mathrm{a})$ stress-strain curve and (b) generation of excess pore water pressures. 

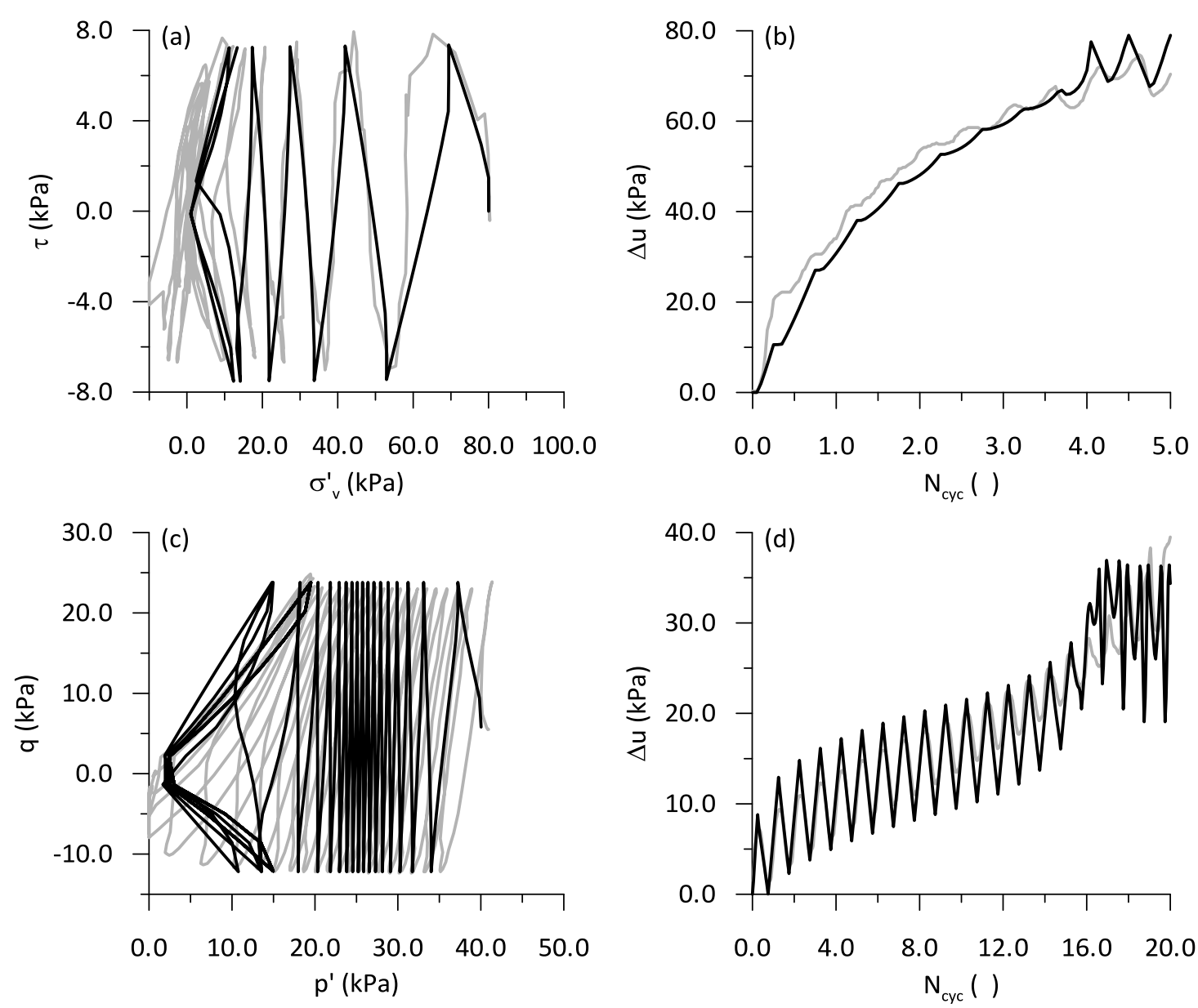

Figure 16: Simulation of two cyclic tests performed on samples of Nevada Sand [31] - (a) stress path and (b) generation of excess pore water pressures measured on a Direct Shear test $(\mathrm{Dr}=$ $45.9 \%, \sigma_{v, 0}^{\prime}=80 \mathrm{kPa}$ ); (c) stress path and (b) generation of excess pore water pressures measured on a Cyclic Triaxial test $\left(\mathrm{Dr}=62.0 \%, p_{0}^{\prime}=40 \mathrm{kPa}\right.$ and $\left.q_{0}=5.8 \mathrm{kPa}\right)$. 
Table 1: Summary of the original formulation of the model proposed by Papadimitriou and Bouckovalas [5].

\section{Description}

Equation

\section{Elastic behaviour}

Small-strain shear modulus

Tangent shear modulus

(2a)

(1) $G_{\text {max }}=\frac{B \cdot p_{\text {ref }}^{\prime}}{0.3+0.7 \cdot e^{2}} \cdot \sqrt{\frac{p^{\prime}}{p_{\text {ref }}^{\prime}}}$

$$
B, p_{\text {ref }}^{\prime}
$$$$
G_{\text {tan }}=\frac{G_{\text {max }}}{1+\kappa \cdot\left(\frac{1}{a_{1}}-1\right) \cdot\left(\frac{\chi_{\text {ref }}^{r}}{N \cdot \eta_{1}}\right)^{\kappa-1}}
$$

(2b) $\eta_{1}=a_{1} \cdot\left(\frac{G_{\max }^{S R}}{p^{\prime S R}}\right) \cdot \gamma_{1}$

Limit tangent shear modulus

(3) $G_{\text {tan }} \geq \frac{G_{\max }}{1+\kappa \cdot\left(\frac{1}{a_{1}}-1\right)}$

Tangent bulk modulus

(4) $K_{\text {tan }}=\frac{2 \cdot(1+v)}{3 \cdot(1-2 \cdot v)} \cdot G_{\text {tan }}$

$v$

\section{Model surfaces}

\begin{tabular}{|c|c|c|c|}
\hline Critical State Line & (5) & $e_{C S}=\left(e_{C S}\right)_{r e f}-\lambda \cdot \ln \left(\frac{p^{\prime}}{p_{r e f}^{\prime}}\right)$ & $\left(e_{C S}\right)_{r e f}, \lambda$ \\
\hline Critical State Surface & (6) & $\sqrt{3} \cdot \overline{J_{2}}=g(\theta, c) \cdot M_{c}^{c} \cdot p^{\prime}$ with $c=M_{e}^{c} / M_{c}^{c}$ & $M_{e}^{c}, M_{c}^{c}$ \\
\hline Dilatancy Surface & (7) & $\sqrt{3} \cdot \overline{J_{2}}=g(\theta, c) \cdot M_{c}^{d} \cdot p^{\prime}=g(\theta, c) \cdot\left(M_{c}^{c}+k_{c}^{d} \cdot \psi\right) \cdot p^{\prime}$ & $k_{c}^{d}$ \\
\hline \multirow[t]{2}{*}{ Bounding Surface } & (8) & $\sqrt{3} \cdot \overline{J_{2}}=g(\theta, c) \cdot M_{c}^{b} \cdot p^{\prime}=g(\theta, c) \cdot\left(M_{c}^{c}+k_{c}^{b} \cdot\langle-\psi\rangle\right) \cdot p^{\prime}$ & $k_{c}^{b}$ \\
\hline & (9a) & $g(\theta, c)=\frac{2 \cdot c}{i_{1}(\theta, c)}-i_{2}(\theta, c)$ & \\
\hline \multirow[t]{2}{*}{$\begin{array}{l}\text { Shape in the deviatoric } \\
\text { plane }\end{array}$} & (9b) & $i_{1}(\theta, c)=\frac{1+c}{2}-\frac{1-c}{2} \cdot \cos \left(3 \cdot \theta+\frac{\pi}{2}\right)$ & \\
\hline & (9c) & $i_{2}(\theta, \mathrm{c})=\frac{1+\mathrm{c}}{2}+\frac{1-\mathrm{c}}{2} \cdot \cos \left(3 \cdot \theta+\frac{\pi}{2}\right)$ & \\
\hline Yield surface & $(10)$ & $F=\sqrt{\left(\boldsymbol{s}-p^{\prime} \cdot \boldsymbol{\alpha}\right):\left(\boldsymbol{s}-p^{\prime} \cdot \boldsymbol{\alpha}\right)}-\sqrt{2 / 3} \cdot m \cdot p^{\prime}=0$ & $m$ \\
\hline \multirow[t]{2}{*}{ Gradient of the yield surface } & $(11 \mathrm{a})$ & $\frac{\partial \mathbf{F}}{\partial \sigma^{\prime}}=\mathbf{n}-\frac{V}{3} \cdot \mathbf{I}_{3}$ & \\
\hline & $(11 b)$ & $\mathbf{n}+\sqrt{2 / 3} \cdot m$ & \\
\hline
\end{tabular}

\section{Plastic behaviour}

Flow rule

(12) $\frac{\mathbf{\partial P}}{\mathbf{\partial \boldsymbol { \sigma } ^ { \prime }}}=\mathbf{n}+\frac{A_{0} \cdot d^{d}}{3} \cdot \mathbf{I}_{\mathbf{3}}$

(13a) $A=p^{\prime} \cdot h_{b} \cdot h_{f} \cdot d^{b}$

Hardening modulus

$$
h_{b}=h_{0} \cdot\left(\frac{p^{\prime}}{p_{\text {ref }}^{\prime}}\right)^{\mu-1} \cdot \frac{\left|d^{b}\right|}{\left\langle d_{\text {ref }}^{b}-\left|d^{b}\right|\right\rangle}
$$




$$
\begin{aligned}
& \text { (13c) } d_{r e f}^{b}=\sqrt{2 / 3} \cdot\left(\left(g(\theta, \mathrm{c}) \cdot M_{c}^{b}-m\right)+\left(g(\theta+\pi, \mathrm{c}) \cdot M_{c}^{b}-m\right)\right) \\
& \text { (13d) } h_{f}=\frac{1+\left\langle f_{p}\right\rangle^{2}}{1+\langle\mathbf{f}: \mathbf{n}\rangle}
\end{aligned}
$$

\section{Hardening rules}

Axis of yield surface

(14) $\Delta \boldsymbol{\alpha}=\langle\Lambda\rangle \cdot h_{b} \cdot h_{f} \cdot\left(\boldsymbol{\alpha}^{\mathbf{b}}-\boldsymbol{\alpha}\right)$

(15a) $\Delta f_{p}=H \cdot \Delta \varepsilon_{v o l}^{p}$

(15b) $\Delta \mathbf{f}=-H \cdot\left\langle-\Delta \varepsilon_{v o l}^{p}\right\rangle \cdot[C \cdot \mathbf{n}+\mathbf{f}]$

Fabric tensor
(15c) $H=H_{0} \cdot\left(\frac{\sigma_{1,0}^{\prime}}{p_{\text {ref }}}\right)^{-\zeta} \cdot\left\langle-\psi_{0}\right\rangle$
$H_{0},-\zeta$
(15d) $\quad C=\max \left|f_{p}\right|^{2}$ 
Table 2: Parameters proposed by Papadimitriou and Bouckovalas [5] for Nevada sand.

\begin{tabular}{|c|c|c|}
\hline Parameter & Description & Value \\
\hline \multicolumn{3}{|l|}{ Critical state line } \\
\hline$p_{r e f}^{\prime}$ & Reference pressure & $98.1 \mathrm{kPa}$ \\
\hline$\left(e_{c s}\right)_{r e f}$ & Void ratio at $p^{\prime}=p_{\text {ref }}^{\prime}$ & 0.809 \\
\hline$\lambda$ & Slope of the CSL & 0.022 \\
\hline \multicolumn{3}{|l|}{ Surface parameters } \\
\hline$M_{c}^{c}$ & Critical state strength in triaxial compression $\left(q / p^{\prime}\right)$ & 1.25 \\
\hline$M_{e}^{c}$ & Critical state strength in triaxial extension $\left(q / p^{\prime}\right)$ & 0.90 \\
\hline$k_{c}^{b}$ & Effect of $\psi$ on the position of the bounding surface & 1.45 \\
\hline$k_{c}^{d}$ & Effect of $\psi$ on the position of the dilatancy surface & 0.30 \\
\hline$A_{0}$ & Dilatancy constant & 2.10 \\
\hline$m$ & Radius of yield surface & 0.065 \\
\hline \multicolumn{3}{|l|}{ Nonlinear elasticity } \\
\hline$B$ & Elastic shear modulus constant & 520.0 \\
\hline$a_{1}$ & Determines $G_{\min } / G_{\max }$ & 0.67 \\
\hline$\kappa$ & Controls nonlinearity of degradation of $G$ & 2.00 \\
\hline$\gamma_{1}$ & Strain limit for degradation of shear modulus & $2.5 \times 10^{-4}$ \\
\hline$v$ & Poisson's ratio & 0.31 \\
\hline \multicolumn{3}{|l|}{ Hardening modulus } \\
\hline$h_{0}$ & Plastic modulus constant & 5000.0 \\
\hline$\mu$ & Effect of mean effective stress & 1.0 \\
\hline \multicolumn{3}{|l|}{ Fabric tensor } \\
\hline$H_{0}$ & Fabric index constant & 68000.0 \\
\hline$\zeta$ & Effect of principal stress on fabric index & 1.0 \\
\hline
\end{tabular}


Table 3: Parameters describing the tested shapes for the Critical State Line.

\begin{tabular}{ccc}
\hline Parameter & $\begin{array}{c}\text { Linear shape } \\
\text { (Papadimitriou and Bouckovalas [5]) }\end{array}$ & $\begin{array}{c}\text { Power law } \\
\text { (Ling and Yang [28]) }\end{array}$ \\
\hline$\left(e_{C S}\right)_{\text {ref }}$ & 0.8090 & 0.8430 \\
$\lambda$ & 0.0220 & 0.0287 \\
$\xi$ & - & 0.7000 \\
$p_{\text {ref }}^{\prime}$ & $98.1 \mathrm{kPa}$ & $101.3 \mathrm{kPa}$
\end{tabular}


Table 4: Summary of the modified formulation of the model.

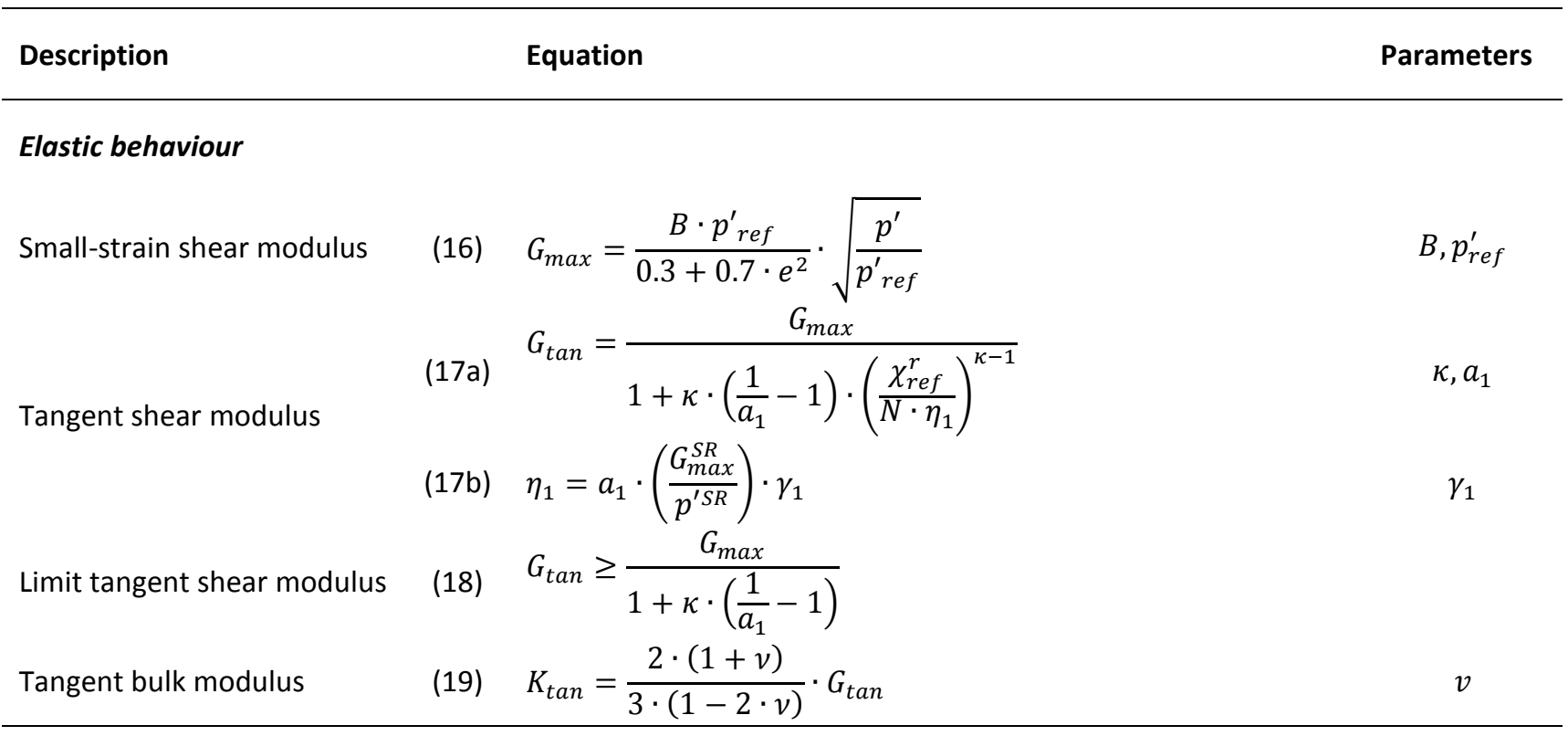

\section{Model surfaces}

\begin{tabular}{|c|c|c|c|}
\hline Critical State Line & (20) & $e_{C S}=\left(e_{C S}\right)_{r e f}-\lambda \cdot\left(\frac{p^{\prime}}{p^{\prime}{ }_{r e f}}\right)^{\xi}$ & $\left(e_{C S}\right)_{r e f}, \lambda, \xi$ \\
\hline Critical State Surface & $(21)$ & $\sqrt{3} \cdot \overline{J_{2}}=g(\theta, c) \cdot M_{c}^{c} \cdot p^{\prime}$ with $c=M_{e}^{c} / M_{c}^{c}$ & $M_{e}^{c}, M_{c}^{c}$ \\
\hline Dilatancy Surface & $(22)$ & $\sqrt{3} \cdot \overline{J_{2}}=g(\theta, c) \cdot M_{c}^{d} \cdot p^{\prime}=g(\theta, c) \cdot\left(M_{c}^{c}+k_{c}^{d} \cdot \psi\right) \cdot p^{\prime}$ & $k_{c}^{d}$ \\
\hline Bounding Surface & $(23)$ & $\sqrt{3} \cdot \overline{J_{2}}=g(\theta, c) \cdot M_{c}^{b} \cdot p^{\prime}=g(\theta, c) \cdot\left(M_{c}^{c}+k_{c}^{b} \cdot\langle-\psi\rangle\right) \cdot p^{\prime}$ & $k_{c}^{b}$ \\
\hline \multirow{3}{*}{$\begin{array}{l}\text { Shape in the deviatoric } \\
\text { plane }\end{array}$} & $(24 a)$ & $g(\theta, c)=\frac{2 \cdot c}{i_{1}(\theta, c)}-i_{2}(\theta, c)$ & \\
\hline & $(24 b)$ & $i_{1}(\theta, c)=\frac{1+c}{2}-\frac{1-c}{2} \cdot \cos \left(3 \cdot \theta+\frac{\pi}{2}\right)$ & \\
\hline & $(24 c)$ & $i_{2}(\theta, \mathrm{c})=\frac{1+\mathrm{c}}{2}+\frac{1-\mathrm{c}}{2} \cdot \cos \left(3 \cdot \theta+\frac{\pi}{2}\right)$ & \\
\hline Primary yield surface & $(25)$ & $F_{1}=\sqrt{\left(s-p^{\prime} \cdot \alpha\right):\left(s-p^{\prime} \cdot \alpha\right)}-\sqrt{2 / 3} \cdot m \cdot p^{\prime}=0$ & $m$ \\
\hline \multirow{2}{*}{$\begin{array}{l}\text { Gradient of the primary } \\
\text { yield surface }\end{array}$} & (26a) & $\frac{\partial \mathbf{F}_{\mathbf{1}}}{\partial \boldsymbol{\sigma}^{\prime}}=\mathbf{n}-\frac{V}{3} \cdot \mathbf{I}_{\mathbf{3}}$ & \\
\hline & $(26 b)$ & $V=\boldsymbol{\alpha}: \mathbf{n}+\sqrt{2 / 3} \cdot m$ & \\
\hline Secondary yield surface & $(27)$ & $F_{2}=p_{Y S}^{\prime}-p^{\prime}=0$ & $p_{Y S}^{\prime}$ \\
\hline $\begin{array}{l}\text { Gradient of the secondary } \\
\text { yield surface }\end{array}$ & (28) & $\frac{\partial F_{2}}{\partial \sigma^{\prime}}=I_{3}$ & \\
\hline
\end{tabular}

Plastic behaviour - primary yield surface

Flow rule

Hardening modulus
(29) $\frac{\partial \mathbf{P}_{\mathbf{1}}}{\mathbf{\partial \boldsymbol { \sigma } ^ { \prime }}}=\mathbf{n}+\frac{A_{0} \cdot d^{d}}{3} \cdot \mathbf{I}_{\mathbf{3}}$

(30a) $A=p^{\prime} \cdot h_{e} \cdot h_{g} \cdot h_{b} \cdot h_{f} \cdot d^{b}$
$A_{0}$ 

(30b) $h_{e}=h_{0} \cdot(1-\gamma) \cdot e \geq h_{0} \cdot(1-\gamma) \cdot e_{\max }$
$h_{0}, \gamma, e_{\max }$
(30c) $h_{g}=G_{\text {tan }}^{\alpha}$
$\alpha$
(30d) $\quad h_{b}=\left(\frac{p^{\prime}}{p_{r e f}^{\prime}}\right)^{\mu-1} \cdot\left(\frac{\left|d^{b}\right|}{\left\langle d_{r e f}^{b}-\left|d^{b}\right|\right\rangle}\right)^{\beta+1}$
(30e) $\quad d_{r e f}^{b}=\sqrt{2 / 3} \cdot\left(\left(g(\theta, c) \cdot M_{c}^{b}-m\right)+\left(g(\theta+\pi, \mathrm{c}) \cdot M_{c}^{b}-m\right)\right)$
(30f) $h_{f}=\frac{1+\left\langle f_{p}\right\rangle^{2}}{1+\langle\mathbf{f}: \mathbf{n}\rangle}$

\section{Plastic behaviour - secondary yield surface}
Flow rule
(31) $\frac{\partial P_{2}}{\partial \sigma^{\prime}}=I_{3}$
Hardening modulus
(32) $A_{2}=0.0$

\section{Hardening rules}

Axis of primary yield surface

(33) $\Delta \boldsymbol{\alpha}=\left\langle\Lambda_{1}\right\rangle \cdot h_{e} \cdot h_{g} \cdot h_{b} \cdot h_{f} \cdot\left(\boldsymbol{\alpha}^{\mathbf{b}}-\boldsymbol{\alpha}\right)$

(34a) $\Delta f_{p}=H \cdot \Delta \varepsilon_{v o l}^{p}$

(34b) $\Delta \mathbf{f}=-H \cdot\left\langle-\Delta \varepsilon_{v o l}^{p}\right\rangle \cdot[C \cdot \mathbf{n}+\mathbf{f}]$

Fabric tensor

$$
\begin{aligned}
& \text { (34c) } \quad H=H_{0} \cdot\left(\frac{\sigma_{1,0}^{\prime}}{p_{\text {ref }}^{\prime}}\right)^{-\zeta} \cdot\left\langle-\psi_{0}\right\rangle \\
& \text { (34d) } \quad C=\max \left|f_{p}\right|^{2}
\end{aligned}
$$$$
H_{0},-\zeta
$$ 
Table 5: Parameters for Nevada sand using the modified formulation [21].

\begin{tabular}{cc}
\hline Parameter & Value \\
\hline$p_{\text {ref }}^{\prime}$ & \\
$\left(e_{c S}\right)_{\text {ref }}$ & $100.0 \mathrm{kPa}$ \\
$\lambda$ & 0.887 \\
$\xi$ & 0.079 \\
\hline
\end{tabular}

Surface parameters

$\begin{array}{cc}M_{c}^{c} & 1.290 \\ M_{e}^{c} & 0.900 \\ k_{c}^{b} & 2.180 \\ k_{c}^{d} & 2.350 \\ A_{0} & 1.460 \\ m & 0.065 \\ p_{Y S}^{\prime} & 1.0 \mathrm{kPa}\end{array}$

Nonlinear elasticity

$\begin{array}{cc}B & 518.6 \\ a_{1} & 0.300 \\ \kappa & 2.000 \\ \gamma_{1} & 6.5 \times 10^{-4} \\ v & 0.200\end{array}$

Hardening modulus

$\begin{array}{cc}h_{0} & 0.613 \\ \gamma & 1.214 \\ e_{\max } & 0.818 \\ \alpha & 1.000 \\ \beta & 0.000 \\ \mu & 1.500\end{array}$

Fabric tensor

$H_{0} \quad 12239.4$

$\zeta \quad 1.590$ 


\section{Appendix A: Calculation of plastic strains and formulation of the elasto-plastic matrix}

The formulation of the constitutive model described in this paper has been presented in generalised stress space, introducing all quantities as tensors. However, as these are symmetrical, only the terms on and above the diagonal need to be stored. For example, the effective stress $\left(\boldsymbol{\sigma}^{\prime}\right)$, strain $(\boldsymbol{\varepsilon})$ and second order identity $\left(\mathbf{I}_{3}\right)$ tensors can be represented by the following vectors:

$$
\begin{aligned}
\boldsymbol{\sigma}^{\prime} & =\left\{\begin{array}{lllllll}
\sigma_{x}^{\prime} & \sigma_{y}^{\prime} & \tau_{x y} & \sigma_{z}^{\prime} & \tau_{x z} & \tau_{y z}
\end{array}\right\}^{T} \\
\boldsymbol{\varepsilon} & =\left\{\begin{array}{lllllll}
\varepsilon_{x} & \varepsilon_{y} & \gamma_{x y} & \varepsilon_{z} & \gamma_{x z} & \gamma_{y z}
\end{array}\right\}^{T} \\
\boldsymbol{I}_{3} & =\left\{\begin{array}{lllllll}
1.0 & 1.0 & 0.0 & 1.0 & 0.0 & 0.0
\end{array}\right\}^{T}
\end{aligned}
$$

Note that italic has been used to distinguish vectorial from tensorial quantities. Similarly, this notation can be used for the gradients of the yield and plastic potential surfaces:

$$
\begin{aligned}
\frac{\boldsymbol{\partial F}}{\boldsymbol{\partial \boldsymbol { \sigma } ^ { \prime }}} & =\left\{\begin{array}{llllll}
\frac{\partial F}{\partial \sigma_{x}^{\prime}} & \frac{\partial F}{\partial \sigma_{y}^{\prime}} & \frac{\partial F}{\partial \tau_{x y}} & \frac{\partial F}{\partial \sigma_{z}^{\prime}} & \frac{\partial F}{\partial \tau_{x z}} & \frac{\partial F}{\partial \tau_{y z}}
\end{array}\right\}^{\boldsymbol{T}} \\
\frac{\boldsymbol{\partial P}}{\boldsymbol{\partial \boldsymbol { \sigma } ^ { \prime }}} & =\left\{\begin{array}{llllll}
\frac{\partial P}{\partial \sigma_{x}^{\prime}} & \frac{\partial P}{\partial \sigma_{y}^{\prime}} & \frac{\partial P}{\partial \tau_{x y}} & \frac{\partial P}{\partial \sigma_{z}^{\prime}} & \frac{\partial P}{\partial \tau_{x z}} & \frac{\partial P}{\partial \tau_{y z}}
\end{array}\right\}^{\boldsymbol{T}}
\end{aligned}
$$

The plastic multiplier, can then be determined using:

$$
\Lambda=\frac{\frac{\partial F^{T}}{\partial \sigma^{\prime}} \cdot \mathbf{D} \cdot \Delta \varepsilon}{\frac{\partial F^{T}}{\partial \sigma^{\prime}} \cdot \mathbf{D} \cdot \frac{\partial P}{\partial \sigma^{\prime}}+A}
$$

where $\mathbf{D}$ is the elastic constitutive matrix:

$$
\mathbf{D}=\left[\begin{array}{cccccc}
K_{\text {tan }}+\frac{4}{3} \cdot G_{\text {tan }} & K_{\text {tan }}-\frac{2}{3} \cdot G_{\text {tan }} & 0 & K_{\text {tan }}-\frac{2}{3} \cdot G_{\text {tan }} & 0 & 0 \\
K_{\text {tan }}-\frac{2}{3} \cdot G_{\text {tan }} & K_{\text {tan }}+\frac{4}{3} \cdot G_{\text {tan }} & 0 & K_{\text {tan }}-\frac{2}{3} \cdot G_{\text {tan }} & 0 & 0 \\
0 & 0 & G_{\text {tan }} & 0 & 0 & 0 \\
K_{\text {tan }}-\frac{2}{3} \cdot G_{\text {tan }} & K_{\text {tan }}-\frac{2}{3} \cdot G_{\text {tan }} & 0 & K_{\text {tan }}+\frac{4}{3} \cdot G_{\text {tan }} & 0 & 0 \\
0 & 0 & 0 & 0 & G_{\text {tan }} & 0 \\
0 & 0 & 0 & 0 & 0 & G_{\text {tan }}
\end{array}\right]
$$

and $A$ is the hardening modulus:

$$
A=-\frac{1}{\Lambda} \cdot \frac{\partial F^{T}}{\partial k} \cdot \Delta k
$$

In the expression above, $\Delta \boldsymbol{k}$ designates the incremental vector of hardening parameters. The incremental plastic strain and incremental stress vectors can then be determined using:

$$
\begin{gathered}
\Delta \varepsilon^{p}=\Lambda \cdot \frac{\partial P}{\partial \sigma^{\prime}} \\
\Delta \sigma^{\prime}=\mathrm{D} \cdot \Delta \varepsilon-\Lambda \cdot \mathrm{D} \cdot \frac{\partial P}{\partial \sigma^{\prime}}
\end{gathered}
$$

while the elasto-plastic matrix can be calculated by: 


$$
\mathbf{D}^{\mathrm{ep}}=\mathrm{D}-\frac{\mathbf{D} \cdot \frac{\partial P}{\partial \sigma^{\prime}} \cdot \frac{\partial F^{T}}{\partial \sigma^{\prime}} \cdot \mathbf{D}}{\frac{\partial F^{T}}{\partial \sigma^{\prime}} \cdot \mathbf{D} \cdot \frac{\partial P}{\partial \sigma^{\prime}}+A}
$$




\section{Appendix B: Elasto-plasticity when two yield surfaces are simultaneously active}

When a constitutive model employs two separate yield surfaces, it is possible for the stress point to activate both simultaneously, thus requiring the equations which govern plasticity, which were introduced in Appendix A, to be adequately altered [20]. In the following description, the vector form as presented in Appendix A, was used for all quantities.

If the stress point be located on both yield surfaces, the plastic component of the incremental strain vector can be further divided into incremental plastic strains associated with each of the two yield surfaces, $\Delta \varepsilon_{1}^{p}$ and $\Delta \varepsilon_{2}^{p}$ :

$$
\Delta \varepsilon=\Delta \varepsilon^{e}+\Delta \varepsilon_{1}^{p}+\Delta \varepsilon_{2}^{p}
$$

As presented in Appendix A, the plastic strains can be evaluated by:

$$
\begin{aligned}
& \Delta \varepsilon_{1}^{p}=\Lambda_{1} \cdot \frac{\partial P_{1}}{\partial \sigma^{\prime}} \\
& \Delta \varepsilon_{2}^{p}=\Lambda_{2} \cdot \frac{\partial P_{2}}{\partial \sigma^{\prime}}
\end{aligned}
$$

Therefore, the stress increment $\Delta \sigma^{\prime}$ can be determined by:

$$
\Delta \sigma^{\prime}=\mathbf{D} \cdot \Delta \varepsilon-\Lambda_{1} \cdot \mathbf{D} \cdot \frac{\partial P_{1}}{\partial \sigma^{\prime}}-\Lambda_{2} \cdot \mathbf{D} \cdot \frac{\partial P_{2}}{\partial \sigma^{\prime}}
$$

Since the stress state satisfies both yield surfaces, $F_{1}=0$ and $F_{2}=0$ must be verified. Using the chain rule of differentiation on the consistency condition, which states that $\Delta F_{1}=\Delta F_{2}=0$, results in:

$$
\begin{aligned}
& \Delta F_{1}=\frac{\partial F_{1}{ }^{T}}{\partial \sigma^{\prime}} \cdot \Delta \sigma^{\prime}+\frac{\partial F_{1}{ }^{T}}{\partial k_{1}} \cdot \Delta k_{1} \\
& \Delta F_{2}=\frac{\partial F_{2}{ }^{T}}{\partial \sigma^{\prime}} \cdot \Delta \sigma^{\prime}+\frac{\partial F_{2}{ }^{T}}{\partial k_{2}} \cdot \Delta k_{2}
\end{aligned}
$$

where $\boldsymbol{k}_{\boldsymbol{i}}$ are the hardening parameters associated to surface $i$. Substituting Eq. 66 into Eq. 67 and Eq. 68 gives:

$$
\begin{aligned}
& \Delta F_{1}=\frac{\partial F_{1}{ }^{T}}{\partial \sigma^{\prime}} \cdot \mathbf{D} \cdot \Delta \varepsilon-\Lambda_{1} \cdot \frac{\partial F_{1}{ }^{T}}{\partial \sigma^{\prime}} \cdot \mathbf{D} \cdot \frac{\partial P_{1}}{\partial \sigma^{\prime}} \\
& -\Lambda_{2} \cdot \frac{\partial F_{1}{ }^{T}}{\partial \sigma^{\prime}} \cdot \mathbf{D} \cdot \frac{\partial P_{2}}{\partial \sigma^{\prime}}-\Lambda_{1} \cdot A_{1}=0 \\
& \Delta F_{2}=\frac{\partial F_{2}{ }^{T}}{\partial \sigma^{\prime}} \cdot \mathbf{D} \cdot \Delta \varepsilon-\Lambda_{1} \cdot \frac{\partial F_{2}}{\partial \sigma^{\prime}} \cdot \mathbf{D} \cdot \frac{\partial P_{1}}{\partial \sigma^{\prime}} \\
& -\Lambda_{2} \cdot \frac{\partial F_{2}{ }^{T}}{\partial \sigma^{\prime}} \cdot \mathbf{D} \cdot \frac{\partial P_{2}}{\partial \sigma^{\prime}}-\Lambda_{2} \cdot A_{2}=0
\end{aligned}
$$

where, as previously presented: 


$$
\begin{aligned}
& A_{1}=-\frac{1}{\Lambda_{1}} \cdot \frac{\partial F_{1}{ }^{T}}{\partial k_{1}} \cdot \Delta k_{1} \\
& A_{2}=-\frac{1}{\Lambda_{2}} \cdot \frac{\partial F_{2}{ }^{T}}{\partial k_{2}} \cdot \Delta k_{2}
\end{aligned}
$$

Eq. 69 and Eq. 70 can be rewritten in the following form:

$$
\begin{aligned}
& \Lambda_{1} \cdot L_{11}+\Lambda_{2} \cdot L_{12}=T_{1} \\
& \Lambda_{2} \cdot L_{21}+\Lambda_{2} \cdot L_{22}=T_{2}
\end{aligned}
$$

where

$$
\begin{aligned}
& L_{11}=\frac{\partial \boldsymbol{F}_{\mathbf{1}}{ }^{\boldsymbol{T}}}{\partial \sigma^{\prime}} \cdot \mathbf{D} \cdot \frac{\partial \boldsymbol{P}_{\mathbf{1}}}{\boldsymbol{\partial \sigma ^ { \prime }}}+A_{1} \\
& L_{22}=\frac{\partial \boldsymbol{F}_{\mathbf{2}}{ }^{\boldsymbol{T}}}{\boldsymbol{\partial \sigma ^ { \prime }}} \cdot \mathbf{D} \cdot \frac{\partial \boldsymbol{P}_{\mathbf{2}}}{\boldsymbol{\partial \sigma ^ { \prime }}}+A_{2} \\
& L_{12}=\frac{\partial F_{\mathbf{1}}{ }^{T}}{\partial \sigma^{\prime}} \cdot \mathbf{D} \cdot \frac{\partial P_{2}}{\partial \sigma^{\prime}} \\
& L_{21}=\frac{\partial F_{2}{ }^{T}}{\partial \sigma^{\prime}} \cdot \mathbf{D} \cdot \frac{\partial P_{1}}{\partial \sigma^{\prime}} \\
& T_{1}=\frac{\partial F_{\mathbf{1}}{ }^{T}}{\partial \sigma^{\prime}} \cdot \mathbf{D} \cdot \Delta \varepsilon \\
& T_{2}=\frac{\partial \boldsymbol{F}_{\mathbf{2}}{ }^{\boldsymbol{T}}}{\boldsymbol{\partial \sigma ^ { \prime }}} \cdot \mathbf{D} \cdot \Delta \boldsymbol{\varepsilon}
\end{aligned}
$$

The system of linear equations composed of Eq. 73 and Eq. 74 can be solved simultaneously since $\Lambda_{1}$ and $\Lambda_{2}$ are the only unknowns, resulting in:

$$
\begin{aligned}
& \Lambda_{1}=\frac{L_{22} \cdot T_{1}-L_{12} \cdot T_{2}}{L_{11} \cdot L_{22}-L_{12} \cdot L_{21}} \\
& \Lambda_{2}=\frac{L_{11} \cdot T_{2}-L_{21} \cdot T_{1}}{L_{11} \cdot L_{22}-L_{12} \cdot L_{21}}
\end{aligned}
$$

The elasto-plastic constitutive matrix is then determined by:

$$
\mathrm{D}^{\mathrm{ep}}=\mathrm{D}-\frac{\mathrm{D}}{\Omega} \cdot\left[\frac{\partial P_{1}}{\partial \sigma^{\prime}} \cdot b_{1}^{T}+\frac{\partial P_{2}}{\partial \sigma^{\prime}} \cdot b_{2}^{T}\right] \cdot \mathrm{D}
$$

where

$$
\Omega=L_{11} \cdot L_{22}-L_{12} \cdot L_{21}
$$

and

$$
\begin{aligned}
& \boldsymbol{b}_{\mathbf{1}}^{\boldsymbol{T}}=L_{22} \cdot \frac{\partial \boldsymbol{F}_{\mathbf{1}}}{\partial \boldsymbol{\sigma}^{\prime}}-L_{12} \cdot \frac{\partial \boldsymbol{F}_{\mathbf{2}}}{\partial \boldsymbol{\sigma}^{\prime}} \\
& \boldsymbol{b}_{2}^{T}=L_{11} \cdot \frac{\partial \boldsymbol{F}_{\mathbf{2}}}{\partial \boldsymbol{\sigma}^{\prime}}-L_{21} \cdot \frac{\partial \boldsymbol{F}_{\mathbf{1}}}{\partial \boldsymbol{\sigma}^{\prime}}
\end{aligned}
$$

